\title{
ERGODIC EQUIVALENCE RELATIONS, COHOMOLOGY, AND VON NEUMANN ALGEBRAS. II
}

\author{
BY \\ JACOB FELDMAN ${ }^{1}$ AND CALVIN C. MOORE ${ }^{2}$
}

\begin{abstract}
Let $R$ be a Borel equivalence relation with countable equivalence classes, on the standard Borel space $(X, Q, \mu)$. Let $\sigma$ be a 2-cohomology class on $R$ with values in the torus $T$. We construct a factor von Neumann algebra $M(R, \sigma)$, generalizing the group-measure space construction of Murray and von Neumann [1] and previous generalizations by W. Krieger [1] and G. Zeller-Meier [1].

Very roughly, $\mathbf{M}(R, \sigma)$ is a sort of twisted matrix algebra whose elements are matrices $\left(a_{x y y}\right)$, where $(x, y) \in R$. The main result, Theorem 1 , is the axiomatization of such factors; any factor $M$ with a regular MASA subalgebra $A$, and possessing a conditional expectation from $M$ onto $A$, and isomorphic to $\mathbf{M}(R, \sigma)$ in such a manner that $A$ becomes the "diagonal matrices"; $(R, \sigma)$ is uniquely determined by $M$ and $A$. A number of results are proved, linking invariants and automorphisms of the system $(M, A)$ with those of $(R, \sigma)$. These generalize results of Singer [1] and of Connes [1]. Finally, several results are given which contain fragmentary information about what happens with a single $M$ but two different subalgebras $\mathbf{A}_{1}, \mathbf{A}_{2}$.
\end{abstract}

1. Introduction. This paper is a sequel to Feldman-Moore [1], hereafter referred to as FMI, where we developed a general theory of what we called countable standard equivalence relations and their cohomology. In the present paper we shift the emphasis and develop the connection of all of this with the theory of von Neumann algebras. This connection starts with the Murray-von Neumann [1] group-measure algebra construction, in which one attaches a factor von Neumann algebra to a pair $(G, X)$ consisting of a freely acting ergodic countable group $G$ on a measure space $(X, \mathscr{B}, \mu)$. It has been observed that the von Neumann algebra depends only on the relation $R_{G}$ generated by the group $G$. Thus one feels that one should be able to construct the algebra directly out of a countable standard relation $R$ without mention of $G$ and whether or not there is a freely acting group. In fact, Krieger [1] did eliminate the hypothesis of freeness in the group-measure construction, but by going over to the relation and forgetting about the group we can make the construction in a rather more transparent and immediate fashion. The von

Received by the editors November $18,1975$.

AMS (MOS) subject classifications (1970). Primary 46L10; Secondary 22D40, 28A65, 46M99.

'Supported in part by National Science Foundation Grant MPS-75-05576.

${ }^{2}$ Supported in part by National Science Foundation Grant MPS-74-19876. 
Neumann algebra $\mathbf{M}(R)$ associated in this way to $R$ appears as the algebra of matrices over the relation $R$; the algebra $B\left(\mathcal{K}_{n}\right)$ of bounded operators on an $n$ dimensional space is the algebra of matrices over the ergodic relation of type $I_{n}$, i.e., the algebra of $n \times n$ matrices. Furthermore, Zeller-Meier [1] showed how to "twist" the group-measure constructions by a two cocycle. This comes out naturally in our context: given $\sigma \in H^{2}(R, \mathbf{T})$ where $\mathbf{T}$ is the circle group, we form the "twisted" algebra of matrices over $R$, denoted by $\mathbf{M}(R, \sigma)$. Our object is to study these algebras and one of our main results, Theorem 1, is a reasonably simple necessary and sufficient condition for an algebra to be isomorphic to one of these. We introduce the notion of a Cartan subalgebra of a von Neumann algebra, motivated by the example of the natural subalgebra of $\mathbf{M}(R, \sigma)$ consisting of the "diagonal" matrices. Our result then is that if a von Neumann algebra $\mathbf{M}$ has a Cartan subalgebra $\mathbf{A}$, then $\mathbf{M}$ is isomorphic to an $\mathbf{M}(R, \sigma)$ in such a manner that $A$ becomes the diagonal matrices. Our definition of Cartan subalgebras agrees with that proposed by Veršik [1]. Since an ergodic relation defines a virtual group, one sees that our results are related to those of P. Hahn [1], who defines the regular representation of a virtual group.

The results above pose all sorts of problems about automorphisms and about conjugacy of Cartan subalgebras, and we have only fragmentary answers.

2. Construction of the von Neumann algebra $M(R, \sigma)$. We turn to the construction and elementary properties of the algebras $M(R, \sigma)$ with $\sigma \in$ $H^{2}(R, \mathbf{T})$ where $R$ is a countable standard relation on $(X, \mathbb{Q}, \mu)$ with $\mu(X)=$ 1. Let $\nu$ be the right counting measure on $R$ defined in Theorem 2 of FMI, and let $\mathcal{H}=L_{2}(R, v)$. We observe that the diagonal $\Delta$ of $X$ in $R$ is a set of measure one and we let $\phi_{0}$ be its characteristic function so that $\phi_{0}$ is a unit vector in $\mathcal{H}$. We select, by Propositions 7.6 and 7.7 of FMI, a cocycle representative $s$ of $\sigma$ which is skew symmetric in its three variables. Finally, let $D$ be the Radon-Nikodym derivative of $\mu$ with respect to $R$ as defined in FMI, so that $D \in Z^{1}\left(R, \mathrm{R}^{+}\right)$.

Definition 2.1. Let $a$ be a complex Borel function on $R$; we say that it is band limited if there is an integer $n$ such that for each $x$ and $y, \mid\{z$ : $a(x, z) \neq 0\}|+|\{z: a(z, y) \neq 0\} \mid \leqslant n$. If $a$ is also bounded we say that it is a finite function or, if necessary to be more precise, a left finite function. (A function $b$ will be right finite if $D(x, y)^{-1 / 2} b(x, y)$ is left finite.)

Proposition 2.1. If $\psi \in \mathscr{C}=L^{2}(R, v)$ and if $a$ is a left finite function and $b$ is right finite, the formulas

$$
L_{a}(\psi)(x, z)=\sum_{y \sim x} a(x, y) \psi(y, z) s(x, y, z),
$$




$$
R_{b}(\psi)(x, z)=\sum_{y \sim x} \psi(x, y) b(y, z) s(x, y, z)
$$

define bounded operators on $\mathcal{H}$.

Proof. Let $B$ be a bound for $a$, and let $A(z)=\{x: a(z, x) \neq 0\}$. Then by definition there is an $n$ so that $A(z)$ has at most $n$ elements, and each $y$ is contained in $A(z)$ for at most $n$ different values of $z$. The sums above are in effect finite sums for each fixed $x$ and $z$; in fact, there are at most $n$ terms, so there is no convergence problem. Now

$$
\int\left|L_{a}(\psi)(x, z)\right|^{2} d \nu(x, y)=\int \sum_{z}\left|L_{a}(\psi)(z, x)\right|^{2} d \mu(x)
$$

by making use of the definition of $\nu$ as right counting measure or equivalently disintegrating $\nu$ with respect to projection to the second coordinate. The integrand above can be estimated above by

$$
\sum_{z}\left(\sum_{y}|a(z, y) \| \psi(y, x)|\right)^{2},
$$

which is no larger than

$$
\sum_{z} B^{2}\left(\sum_{y \in A(z)}|\psi(y, x)|^{2}\right),
$$

which by the Schwarz inequality is dominated by

$$
B^{2} n \sum_{z} \sum_{y \in A(z)}|\psi(y, x)|^{2}
$$

which in turn, by properties of $A(z)$, is dominated by

$$
B^{2} n^{2} \sum_{w}|\psi(w, x)|^{2}
$$

We then conclude that $L_{a}(\psi)$ is in $\mathcal{H}$ and $\left|L_{a}(\psi)\right| \leqslant B n|\psi|$.

In order to treat $R_{a}$ we have to disintegrate the measure $\nu$ with respect to projection to the left factor so that we have to compare right and left counting measures and the Radon-Nikodym derivative $D$ will enter. Specifically

$$
\begin{aligned}
\int\left|R_{b}(\psi)(z, x)\right|^{2} d \nu & =\int \sum_{x}\left|R_{b}(\psi)(z, x)\right|^{2} D(z, x)^{-1} d \mu(z) \\
& <\int \sum_{x}\left|\sum_{y} \psi(z, y) D(y, x)^{-1 / 2} b(y, x)\right|^{2} D(z, y)^{-1} d \mu(z)
\end{aligned}
$$

since $D(z, x)=D(z, y) D(y, x)$; then as before we obtain an estimate for this of the form 


$$
C^{2} n^{2} \int \sum_{y}|\psi(z, y)|^{2} D(z, y)^{-1} d \mu(z)=C^{2} n^{2}\|\psi\|^{2}
$$

where $C$ is uniform bound for $b(y, x) D(y, x)^{-1 / 2}$.

For two band limited functions $a$ and $b$ we define a product $a b(x, z)=$ $\Sigma_{y} a(x, y) b(y, z) s(x, y, z)$. It is clear that $a b$ is also band limited. We define $a^{*}(x, y)=\overline{a(y, x)}$ and $a_{*}(x, y)=\overline{a(y, x)} D(y, x)$.

Proposition 2.2. If $a, b$ are left (right) finite, so is $a b$, and

$$
L_{a} L_{b}=L_{a b}\left(R_{a} R_{b}=R_{a b}\right) \text {. }
$$

If $a$ is left (right) finite, $a^{*}\left(a_{*}\right)$ is left (right) finite and $L_{a}^{*}=L_{a^{*}}\left(R_{a}^{*}=R_{a_{*}}\right)$.

Proof. These routine calculations are omitted.

Definition 2.2. The operators $L_{a}, a$ left finite, form a *-algebra of operators $\mathbf{L}$ with unit; we denote its weak closure by $\mathbf{M}(R, s)$. Similarly the operators $R_{b}$ form a ${ }^{*}$-algebra $\mathbf{R}$ with unit; we denote its weak closure by $\tilde{\mathbf{M}}(R, s)$.

Now if $\phi_{0}$ is the characteristic function of the diagonal, then $\left(L_{a} \phi_{0}\right)(x, z)=$ $a(x, z)$, since $s$ is skew symmetric, and similarly $\left(R_{a} \phi_{0}\right)(x, z)=a(x, z)$.

Proposition 2.3. Any left or right finite function is in $L^{2}(R, v)$, and the set of either is dense. Any right or left bounded function can be written as a finite linear combination of functions of the form $f(x) F(\phi)(x, y)$ where $\phi$ is a partial Borel isomorphism of some subset of $X$ onto another with $\Gamma(\phi) \subset R$, where $F(\phi)$ is the characteristic function of the graph of $\phi^{-1}$ and where $f$ is bounded in the case of left finite functions or $f(x) D\left(x, \phi^{-1}(x)\right)^{-1 / 2}$ is bounded in the case of right finite functions. Any such function of either type may be approximated by ones where $D\left(x, \phi^{-1}(x)\right)$ is bounded above and below.

Proof. The fact that left or right finite functions are in $L^{2}$ is immediate from the comment immediately preceding the proposition. We consider a left finite function and let $E$ be the subset of $R$ where it is nonzero. The band limited condition implies that the inverse image of any point $x \in X$ under projection to either coordinate has at most $n$ points. As in Theorem 1 of FMI, we can find a countable partition $E=\cup_{i} E_{i}$ so the projection to the second coordinate, called $\pi_{r}$, is one-to-one on each $E_{i}$. We may also assume that for $i<j, \pi_{r}\left(E_{i}\right) \supset \pi_{r}\left(E_{j}\right)$, since by a simple but tedious double induction on $i$ and $j$ we in effect add to $E_{i}$ the set $E_{j} \cap \pi_{r}^{-1}\left(E_{i}^{c}\right)$. Once this is done it is evident that there are at most $n E_{i}$ 's. Now we repeat this same argument applied to each $E_{i}$ and the projection $\pi_{l}$ on the first coordinate, to write it as a disjoint union of at most $n$ sets where $\pi_{l}$ is injective on each. Then $E$ is the disjoint union of a finite number (at most $n^{2}$ ) of sets $F_{j}$ such that $\pi_{l}$ and $\pi_{r}$ are injective on each $F_{j}$. But then each $F_{j}$ is the graph of a partial Borel 
isomorphism $\phi_{j}^{-1}$, and since $F_{j} \subset R, \Gamma\left(\phi_{j}\right) \subset R$. Now it is obvious that any bounded Borel function $a$ vanishing off $E$ is a linear combination of functions $f_{j}(x) F\left(\phi_{j}\right)(x, y)$ as desired. If instead $a$ is right finite, $a(x, y) D(x, y)^{-1 / 2}$ is left bounded and so $a$ has the form indicated. Finally, a function of the form $f(x) F(\phi)(x, y)$ can be approximated in $L^{2}$ by $f(x) F(\phi(n))(x, y)$ where $\phi(n)$ is the restriction of $\phi$ to the subset of its domain where $1 / n<$ $D\left(x, \phi^{-1}(x)\right)<n$.

It remains only to prove that the right or left finite functions are dense in $L^{2}$. We do it for left finite functions, and note that any function $g$ perpendicular to them must be perpendicular to all functions of the form $f(x) F(\phi)(x, y)$, where $\phi$ is a partial Borel isomorphism with domain $D(\phi)$. It follows that $g=0$ a.e. on the graph $\Gamma\left(\phi^{-1}\right)$ and since $R$ is a countable union of such sets, $g=0$ a.e. and we are done.

The following variation of the above will be of use shortly.

Proposition 2.4. Every left finite function is a finite linear combination of functions of the form $g(x) F(\psi)$ with $g$ of absolute value one, where $\psi$ is a Borel isomorphism of all of $X$ onto all of $X$ with $\Gamma(\psi) \subset R$.

Proof. It suffices to show the result for any function of the form $F(\phi)$ since any bounded function is a finite linear combination of functions of absolute value one. Assume for the moment that the relation is ergodic and let $A$ be the domain of $\phi$. Then by Proposition 3.3 of FMI, one sees that $\phi$ may be extended to a Borel isomorphism of $X$ onto $X$ with $\Gamma(\phi) \subset R$ if $R$ is finite, and if $R$ is infinite it is easy to see by the same result that we can partition $A=A_{1} \cup A_{2}$ with $\phi_{i}$ the restriction of $\phi$ to $A_{i}$ and find extensions $\psi_{i}, i=1,2$, of $\phi_{i}$ to Borel isomorphisms of $X$ onto $X$. If $R$ is not ergodic it is not hard to extend this result using direct integral decompositions. In any case, such $\psi_{1}$ and $\psi_{2}$ always exist and it is evident that $F(\phi)$ is a linear combination of functions $g_{i} F\left(\phi_{i}\right)$ with $g_{i}$ of absolute value one.

We can now establish some basic properties of the algebras $\mathbf{M}(R, s)$ and $\tilde{\mathbf{M}}(R, s)$.

Proposition 2.5. The algebras $\mathbf{M}(R, s)$ and $\tilde{\mathbf{M}}(R, s)$ are commutants of each other and the vector $\phi_{0}$ (defined above to be the characteristic function of the diagonal) is separating and cyclic for both algebras.

Proof. $\mathbf{L} \phi_{0}\left(\mathbf{R} \phi_{0}\right)$ consists of right (left) finite functions viewed as elements of $L^{2}(R, v)$, so $\phi_{0}$ is a cyclic vector for $\mathbf{L}$ and $\mathbf{R}$, and hence for $\mathbf{M}(R, s)$ and $\tilde{\mathbf{M}}(R, s)$. Then $\phi_{0}$ is separating once we know that these algebras are commutants of each other. To show this we note the $\mathbf{L}$ and $\mathbf{R}$ commute and then invoke the criterion of Rieffel and van Daele [1]; it therefore suffices to show that $\mathcal{W}=\mathbf{L}_{s}\left(\phi_{0}\right)+i \mathbf{R}_{s}\left(\phi_{0}\right)$ is dense in $L^{2}(R, \nu)$ where $\mathbf{L}_{s}$ and $\mathbf{R}_{s}$ are the 
selfadjoint elements of $\mathbf{L}$ and $\mathbf{R}$ respectively. By Proposition 2.3, it suffices to show that any element of $\mathcal{H}$ of the form $f(x) F(\theta)(x, y)$ is in $\mathcal{W}$, where $\theta$ is a partial Borel isomorphism on $X$ with $\Gamma(\theta) \subset R$ with $D\left(x, \theta^{-1}(x)\right)$ bounded above and below.

Since $\mathbf{L}_{s} \cdot \phi_{0}$ consists of (left) finite functions $a$ such that $a(x, y)=\bar{a}(y, x)$, and $i \mathbf{R}_{s} \cdot \phi_{0}$ consists of right finite functions of the form $i b$ where $b(x, y)=\overline{b(y, x)} D(y, x)$, it is an easy, although tedious, matter now to display $f(x) F(\theta)(x, y)$ in the form $a+i b$. We omit the routine details.

Now if $T \in M(R, s)$ then the vector $T \phi_{0}$ in $L^{2}(R, \nu)$ completely determines $T$, and we write $a(T)=T \phi_{0}$. Similarly, if $T \in \tilde{\mathbf{M}}(R, s)=\mathbf{M}(R, s)^{\prime}$, then the vector $T \phi_{0}=b(T)$ determines $T$ entirely.

Proposition 2.6. If $T \in \mathbf{M}(R, s), a=a(T)$ is square integrable for right and left counting measures on $R$, and is bounded by the operator norm of $T$. Moreover, for $\psi \in L^{2}(R, \nu)$,

(1) $(T \psi)(x, z)=\Sigma_{y} a(T)(x, y) \psi(y, z) s(x, y, z)$, so that $T=R_{a(T)}$ in an extended sense. Moreover,

(2) $a\left(T_{1} T_{2}\right)(x, z)=\Sigma_{y} a\left(T_{1}\right)(x, y) a\left(T_{2}\right)(y, z) s(x, y, z)$, and $a\left(T^{*}\right)(x, y)=\overline{a(T)}(y, x)$.

Corresponding results hold for $P$ in $\mathbf{M}(R, s)^{\prime}$ and $b(P)$.

Proof. Clearly $a(T)=T \phi \in L^{2}\left(R, \nu_{r}\right)$ where $\nu=\nu_{r}$ is right counting measure. But now $a\left(T^{*}\right)(x, y)=\overline{a(T)}(y, x)$ for $T \in \mathbf{L}$ by Proposition 2.2 and since $\mathrm{L}$ is dense, this follows immediately for all $T$. Then $\overline{a(T)}(y, x) \in$ $L^{2}\left(R, \nu_{r}\right)$. But this implies that $a(T) \in L^{2}\left(R, \nu_{l}\right)$ where $\nu_{l}$ is left counting measure. Now using the fact that $\mathbf{M}(R, s)$ commutes with $\mathbf{R}$, it is clear that formula (1) of the proposition holds for right finite functions $\psi$, and since $a(T) \in L^{2}\left(R, v_{l}\right)$ it follows that $\Sigma_{y}|a(T)(x, y)|^{2}$ converges for almost all $x$. We can approximate $\psi$ by a sequence of right finite vectors $\psi(n)$ so that $\psi(n)(\cdot, z) \rightarrow \psi(\cdot, z)$ in $l_{2}(R(z))$ for almost all $z$. It follows that the right-hand side of (1) for $\psi(n)$ converges to the right-hand side for $\psi$ for almost all pairs $(x, z)$. Hence the formula holds for $\psi$. We establish formula (2) in a similar fashion. It remains only to show that $a(T)$ is bounded by the norm of $T$. In fact, if $|a(T)|(x, y)>|T|$ on a set $E$ of positive measure, then we may select a partial Borel isomorphism $\theta$ of $X$ with $(\theta(x), x) \in E$ for all $x$ in the domain $A$ of $\theta$. Now let $\psi_{1}$ be the characteristic function of the set $\Delta(A)=\{(x, x)$ : $x \in A\}$, and let $\psi_{2}$ be the characteristic function of $\{(\theta(x), x), x \in A\}$ times $\overline{a(T)}(\theta(x), x) /|a(T)(\theta(x), x)|$. Evidently both $\psi_{1}$ and $\psi_{2}$ have norms equal to $\mu(A)^{1 / 2}$, and

$$
\left(T \psi_{1}, \psi_{2}\right)=\int_{A}|a(T)|(\theta(x), x) d \mu(x)>\|T\|\left\|\psi_{1}\right\|\left\|\psi_{2}\right\|,
$$

a contradiction. 
Thus we have realized the algebras $\mathbf{M}=\mathbf{M}(R, s)$ and its commutant as generalized matrix algebras where the matrix for $T \in \mathbf{M}$ is $a(T)$, a bounded function on $R$. One cannot hope to say exactly which such functions occur; after all, one cannot do this even in the special case when the relation $R$ is $I_{\infty}$, the ergodic relation of type $\mathrm{I}_{\infty}$. For here, $\mathrm{I}_{\infty}=S_{\infty} \times S_{\infty}$ for a discrete set $X=S_{\infty}$, and evidently the algebra $\mathbf{M}\left(\mathrm{I}_{\infty}, 1\right)$ is $\mathbf{B}\left(\mathcal{H}_{\infty}\right)$, the bounded operators on a separable Hilbert space realized as infinite matrices. The feature of twisting by a cocycle does not enter here; this is the case more generally for $\boldsymbol{R}$ hyperfinite, by Theorem 6 of FMI. It is readily verified also that if $G$ is a freely acting group on $X$ and $R=R_{G}$, then $\mathbf{M}\left(R_{G}, 1\right)$ is the result of the group measure construction of von Neumann and Murray. The identification is made by noting that $(g, x) \mapsto(g \cdot x, x)$ is a bijection from $G \times X$ to $R_{G}$. Algebras $\mathrm{M}\left(R_{G}, s\right)$ for a cocycle $s$ can be identified as the algebras of Zeller-Meier [1]. For non freely acting groups these algebras were constructed somewhat differently in Krieger [1]. We note that if we change the cocycle $s$ by a coboundary $\delta c(x, y, z)$, where $c$ is skew symmetric (as it must be if the modified $s$ is to be skew symmetric), then we obtain an isomorphic algebra. Indeed, if $L_{a}^{t}$ denotes the operator with matrix $a$ using the cocycle $t$ and if we define a unitary operator $C$ on $\mathcal{H}$ by $(C \psi)(x, y)=c(x, y) \psi(x, y)$, then $C L_{a}^{s} C^{-1}=L_{c \cdot a}^{(\delta c)(s)}$ where $c \cdot a$ is the pointwise product. Thus $C M(R, s) C^{-1}$ $=\mathbf{M}(R,(\delta c)(s))$, so that the isomorphism type of $\mathbf{M}(R, s)$ depends only on the cohomology class $\sigma$ of $s$ and we write $\mathbf{M}(R, \sigma)$. Additionally, it is appropriate to note for later use what happens if $\delta c=1$.

Proposition 2.7. If $c \in Z^{1}(R, T)$ is a one-cocycle, then $L_{a} \mapsto L_{c \cdot a}$ is a *-automorphism of $\mathbf{M}(R, s)=\mathbf{M}(R, \sigma)$ which we shall denote by $\pi(c)$, and which is spatially implemented by the unitary operator $C$, where $(C \psi)(x, y)=$ $c(x, y) \psi(x, y)$.

The algebra $\mathbf{M}=\mathbf{M}(R, s)$ comes with a separating and cyclic vector $\phi_{0}$, so that one may apply the Tomita-Takesaki construction to obtain the modular automorphism group $\psi_{\ell}$ and anti-unitary $J$ with $\mathbf{J M J}=\mathbf{M}^{\prime}$. We note what these objects are in our context:

Proposition 2.8. The modular operator $\Delta$ is multiplication by $D,(\Delta \psi)(x, y)$ $=D(x, y) \psi(x, y)$, and the natural domain of $\Delta^{1 / 2}$ is $L^{2}\left(R, \nu_{r}\right) \cap L^{2}\left(R, \nu_{l}\right)$, that is, functions square integrable with respect to both right and left counting measure. The modular automorphism group $\psi_{t}$ is given by $\psi_{t}=\pi\left(D^{i t}\right)$ with $D^{i t} \in Z^{1}(R, \mathbb{T})$ using the notation of Proposition 2.7. The anti-unitary $J$ has the form $J \psi(x, y)=\bar{\psi}(y, x) D^{-1 / 2}(y, x)$ and $J L_{a} J=R_{b}$ where $b(x, y)=$ $\bar{a}(y, x) D(y, x)^{-1 / 2}$.

PROOF. The operator $S$ defined by $S\left(L_{a} \phi_{0}\right)=\left(L_{a}\right)^{*} \phi_{0}$ has the form 
$(S \psi)(x, y)=\bar{\psi}(y, x)$ according to the formulas above. It is easy to check that $(J \psi)(x, y)=\bar{\psi}(y, x) D^{-1 / 2}(y, x)$ defines an isometry, and that $(\Delta \psi)(x, y)=$ $D(x, y) \psi(x, y)$ defines a selfadjoint operator. One checks that $S=J \Delta^{1 / 2}$ and it follows that the Tomita-Takesaki $J$ and $\Delta$ are given by these formulas. The statement about the domain of $D^{1 / 2}$ follows from the definition of $D$ as $\left(d \nu_{l} / d \nu_{r}\right)$. The modular automorphism group $\psi_{t}$ is implemented by the operators $\Delta^{i t}$, which are evidently operators of multiplication by the functions $(x, y) \mapsto D(x, y)^{i t}$. Now, $D \in Z^{1}\left(R, \mathbf{R}^{+}\right)$, so $D(x, y)^{i t}$ is in $Z^{1}(R, \mathbf{T})$. The final formula for $J L_{a} J$ is a simple computation.

We will denote by $\omega$ the state of $\mathbf{M}$ defined by the vector $\phi_{0}$. Now, $\mathbf{M}$ has a naturally defined abelian subalgebra consisting of "diagonal" operators. Specifically, if $a \in L^{\infty}(X)$, we view it as a function on $R$, also denoted by $a$, by defining $a(x, y)=a(x)$ if $x=y$ and 0 otherwise, so $a$ is supported on the diagonal $\Delta \subset R$; recall that $\Delta$ is a set of positive measure and $\left.\nu\right|_{\Delta}$ is equivalent to $\mu$. Then $a$ as a function on $R$ is clearly left and right finite and so defines operators $L_{a}$ and $R_{a}$ in $\mathbf{M}$ and $\mathbf{M}^{\prime}$. These are given by $\left(L_{a} \psi\right)(x, y)=$ $a(x) \psi(x, y)$ and $\left(R_{a} \psi\right)(x, y)=\psi(x, y) a(y)$. Thus $\left\{L_{a}\right\}$ forms already an abelian von Neumann subalgebra $A$ of $M$, isomorphic to $L^{\infty}(X)$; and similarly $\left\{R_{a}\right\}$ forms a subalgebra of $\mathbf{M}^{\prime}$ isomorphic to $L^{\infty}(X)$ which we shall denote by $\mathbf{B}$.

Recall that the normalizer $\mathbf{N}(\mathbf{A})$ of a subalgebra consists of the unitary operators $U$ in $M$ such that $U A U^{-1}=A$. Note that $\mathbf{N}(\mathrm{A})$ contains the unitary group $\mathbf{U}(A)$ of $A$, and $U(A)$ is normal in $\mathbf{N}(A)$. We denote the quotient group by W(A), the Weyl group. Recall from Dixmier [1] that an abelian subalgebra $\mathbf{A}$ of $\mathbf{M}$ is regular if $\mathbf{N}(\mathbf{A})$ generates $\mathbf{M}$.

Finally, if $L_{a} \in \mathbf{M}$, we know by Proposition 2.6 that $a$ is bounded; so if $E(a)$ is the restriction of $a$ to the diagonal, then $E(a)$ defines an operator $L_{E(a)}$ in $\mathbf{A}$.

Proposition 2.9. (1) The subalgebra $\mathbf{A}$ of $\mathbf{M}$ is maximal abelian, and likewise the subalgebra $\mathbf{B}$ of $\mathbf{M}^{\prime}$. Moreover, $J L_{a} J=R_{\bar{a}}$ for $L_{a} \in \mathbf{A}$, so that $\mathbf{B}=J \mathbf{A} J$; and $\mathbf{A}$ and JAJ together generate a maximal abelian subalgebra of $\mathbf{B}\left(L^{2}(R)\right)$.

(2) The center of $\mathbf{M}$ consists of $\left\{L_{a}: a \in G(R)\right\}$, where $G(R)$, as defined in $F M I$, is the invariant functions on $R$, so that $\mathbf{M}$ is a factor if and only if $R$ is ergodic.

(3) $\mathbf{A}$ is a regular subalgebra and its Weyl group $\mathbf{W}(\mathrm{A})$ is isomorphic to $G(R)$, the full group of the relation (the bijections $\phi$ on $X$ with $\mu \circ \phi \sim \mu$ and $\phi x \sim x$ a.e.).

(4) The map $E\left(L_{a}\right)=L_{E(a)}$ is a normal faithful conditional expectation of $\mathbf{M}$ onto A. It satisfies the equation $\omega(E(T))=\omega(T)$ where $\omega$ is the state defined by $\phi_{0}$, and $E\left(U T U^{-1}\right)=U E(T) U^{-1}$ for $U \in \mathbf{N}(\mathbf{A})$. 
Proof. (1) The formula $J L_{a} J=R_{\bar{a}}$ is clear for $L_{a} \in \mathrm{A}$. Now A and $J A J$ can be viewed as subalgebras of the algebra $\mathrm{C}$ on $L^{2}(R)$ consisting of multiplications by all bounded functions on $R$ which we may identify as $L^{\infty}(R)$ with $\mathbf{A}$ and $J \mathbf{A} J$ sitting inside as functions depending only on the first, respectively second, coordinate. Since the projections to the coordinates separate points, it follows (cf. Segal [1]) that the images of $\mathbf{A}$ and $J \mathbf{A} J$ in $L^{\infty}(R)$ generate $L^{\infty}(R)$ and hence that $\mathbf{A}$ and $J \mathbf{A} J$ generate $\mathbf{C}$, which is maximal abelian in $\mathbf{B}\left(L^{2}(R)\right)$, proving the last statement in (1). To see that $\mathbf{A}$ is maximal abelian in $\mathbf{M}$, note that if $L_{a}$ commutes with $\mathbf{A}$, then $L_{a}$ commutes with $J \mathbf{A} J \subset \mathbf{M}^{\prime}$ and so commutes with $\mathbf{C}$ and hence is in $\mathbf{C}$ itself. Then $\left(L_{a} \psi\right)(x, y)=c(x, y) \psi(x, y)$ for some function $c$. If we take $\psi=\phi_{0}$, then $L_{a} \phi_{0}=E(c)$, the restriction of $c$ to the diagonal, and since $\phi_{0}$ is separating, $a=E(c)$ and so $L_{a} \in \mathbf{A}, \mathbf{A}$ is maximal abelian in $\mathbf{M}$, and $J \mathbf{A} J$ is maximal abelian in $\mathbf{M}^{\prime}=J \mathbf{M} J$.

(2) Since $\mathbf{A}$ is maximal abelian, the center $\mathbf{Z}$ of $\mathbf{M}$ lies in $\mathbf{A}$ and in $J \mathbf{A} J$. Now since $J T J=T^{*}$ for $T$ in $\mathbf{Z}, \mathbf{Z}$ can be characterized as those $L_{a} \in \mathbf{A}$ such that $L_{a}=R_{a}$. This means, however, that $a(x) \psi(x, y)=\psi(x, y) a(y)$ a.e. for any $\psi$ and one sees immediately that this means $a$ is in $G(R)$.

(3) To see that $\mathbf{A}$ is regular, let $\phi$ be a Borel isomorphism of $X$ onto itself with $\Gamma(\phi) \subset R$ and let $f$ be a function on $X$ of absolute value one, and let $a(f, \phi)(x, y)=f(x) F(\phi)(x, y)$ be a function on $R$ of the type considered in Proposition 2.4 which is equal to $f(x)$ if $x=\phi(y)$ and zero otherwise. For the moment, we need not assume that $|f(x)|=1$. Then the operator $L_{a(f, \phi)}$ has the form

$$
\left(L_{a(f, \phi)}\right)(x, z)=v\left(\phi^{-1}(x), z\right) f(x) s\left(x, \phi^{-1}(x), z\right)
$$

and from this it is clear that $L_{a(f, \phi)}$ is unitary if $|f(x)|=1$. Moreover, by Proposition 2.6, $L_{a(f, \phi)} L_{a(g, \psi)}=L_{a(h, \phi \psi)}$ where

$$
h(x)=f(x) g\left(\phi^{-1}(x)\right) s\left(x, \phi^{-1}(x),(\phi \psi)^{-1}(x)\right),
$$

and it follows that for $|f|=1, L_{a(f, \phi)} L_{a(g, 1)} L_{a(f, \phi)}^{*}=L_{a(h, 1)}$ where $h(x)=$ $g\left(\phi^{-1}(x)\right)$. Since $L_{a(g, 1)}$ is the generic element of $A$, each $L_{a(f, \phi)}$ belongs to the normalizer $\mathbf{N}(\mathbf{A})$ of $\mathbf{A}$, and $L_{a(f, \phi)}$ induces the automorphism $g(x) \mapsto$ $g\left(\phi^{-1}(x)\right)$ of $A \cong L^{\infty}(X)$.

If we knew that $\mathbf{N}(\mathrm{A})$ consisted exactly of the set of all $L_{a(f, \phi)}$, then $L_{a(f, \phi)} \mapsto \phi$ would define, by the formulas above, a homomorphism of $\mathbf{N}(\mathbf{A})$ onto $G(R)$ whose kernel consisted of precisely the elements of the form $L_{a(f, 1)}$, i.e., $\mathbf{U}(\mathrm{A})$. Thus we would have $\mathbf{N}(\mathbf{A}) / \mathbf{U}(\mathbf{A}) \cong G(R)$ as asserted. It remains to show that any $U \in \mathbf{N}(\mathrm{A})$ is of the form $L_{a(f, \phi)}$. But $U L_{a} U^{*}=L_{\theta(a)}$ where $\theta$ is an automorphism of $L^{\infty}(X)$ which is necessarily of the form $\theta(a)(x)=a\left(\phi^{-1}(x)\right)$ for some Borel isomorphism $\phi$ of $X$ onto itself. How- 
ever, $U=L_{b}$ for some function $b \in L^{\infty}(R)$ and so $\theta(a)=b a b^{*}$, and a simple computation shows that

$$
b a b^{*}(x, x)=\sum_{y}|b(x, y)|^{2} a(y)=a\left(\phi^{-1}(x)\right)
$$

holds for any $a \in L^{\infty}(X)$. The only way this could happen is for $b(x, y)=0$ unless $y=\phi^{-1}(x)$, and then $b\left(x, \phi^{-1}(x)\right)=f(x)$ must be of absolute value one. It follows that $\Gamma(\phi) \subset R$ and that $b=a(f, \phi)$ so that $U=L_{a(f, \phi)}$, and this proves (3), and a bit more which we state separately in a moment.

(4) The map $E$ is well defined and evidently $E(1)=1$ and we have $E\left(L_{a} L_{b} L_{c}\right)=L_{a} E\left(L_{b}\right) L_{c}$ when $L_{a}$ and $L_{b}$ are in $\mathbf{A}$, as a result of the formulas of Proposition 2.6. Also, if $b=c c^{*}$, then $b(x, x)=c c^{*}(x, x)=\Sigma_{y}|c(x, y)|^{2}$ so that $E$ is positive and faithful. Now if $L_{a(n)} \rightarrow L_{a}$ strongly, then as $a(n)=L_{a(n)} \phi_{0}$, it follows that $a(n) \rightarrow a$ as elements of $L^{2}$, so $1_{\Delta} a(n) \rightarrow 1_{\Delta} a$ in $L^{2}(X)$. Since $1_{\Delta} a(n) \in L^{\infty}(X)$ and $\left\|1_{\Delta} a(n)\right\|_{\infty} \ldots$ is uniformly bounded, it follows by dominated convergence that $E\left(L_{a(n)}\right) \rightarrow E\left(L_{a}\right)$ strongly. This establishes the normality of $E$. The final two statements of (4) follow by simple calculations.

In the course of the proof of (3) above, we proved a more explicit statement that we will need subsequently.

Proposition 2.10. Every element of $\mathbf{N}(\mathbf{A})$ is of the form $L_{a(f, \phi)}$ with $|f(x)|=$ 1 and with $\phi \in G(R)$. The algebra generated by $\mathbf{N}(\mathbf{A})$ consists of the $L_{b}$ with $b$ left finite.

Proof. The first statement is already proved and the second is a rephrasing of Propositon 2.4.

Note that the operators $L_{a(f, \phi)}$ are the "monomial" operators, which are entirely analogous to the monomial matrices in the matrix presentation of $\mathbf{B}(\mathcal{H C})$. Proposition 2.10 shows somewhat in retrospect our reason for selecting out the left (or right) finite functions as basic objects. Finally, we note that the modular automorphism group $\left\{\psi_{t}\right\}$ associated to the state $\omega$ has the form of multiplication by $D^{i t}$, and hence $\psi_{t}(\mathbf{A})=\mathbf{A}$. It then follows on general principles from Takesaki [1] that there is a faithful, normal, conditional expectation $E$ as in (4) of Proposition 2.9. It is of course useful to have the explicit form of this $E$.

Finally, we note that the asymptotic range of the Radon-Nikodym cocycle $D$ has a natural interpretation (as of course it must) in terms of $\mathbf{M}(R, \sigma)$.

Proposition 2.11. The asymptotic range $r^{*}(D)$ coincides with the Connes invariant $S(\mathrm{M}(R, \sigma))$ for any $\sigma \in H^{2}(R, T)$.

Proof. If $R=R_{G}$ with $G$ free and $\sigma=1$, this is in Connes' thesis [1] and for $G$ not necessarily free, but for $\sigma=1$, the result is in Ghez-Lima-Testard 
[1]. Thus 2.11 is only a routine extension of known facts. Indeed, by Theorem 3.3.1 of Connes [1] and by part (3) and the last statement of part (4) of our Proposition 2.9, it follows that $\lambda \in S(\mathbf{M}(R, \sigma))$ if and only if for all $\varepsilon>0$, and for each nonzero projection $P$ in $\mathbf{A}$, there is a nonzero projection $Q$ in $\mathbf{A}$ with $Q<P$ and some $U \in \mathrm{N}(\mathrm{A})$ such that $U Q U^{*} \leqslant P$, and such that the spectrum of $\rho_{U}^{-1} Q$ in $Q A Q$ lies in the interval $(\lambda-\varepsilon, \lambda+\varepsilon)$ where $\rho_{U}$ is the unbounded operator affiliated to $\mathbf{A}$ such that $\omega\left(U A U^{*}\right)=\omega\left(\rho_{U} A\right)$ for $A \in$ $\mathbf{A}_{+}$. However, if we interpret $\mathbf{A}$ as $L^{\infty}(X), \rho_{U}$ is simply a positive function on $X$ and if we take $U=L_{a(f, \phi)}$ we can identify $\rho$ by Proposition 2.2 as the function $D(\phi(y), y)$. This says that $\lambda \in S(M(R, \sigma))$ iff for all neighborhoods $\Lambda$ of $\lambda$ and all sets $E \subset X$ of positive measure, we can find $F \subset E$ and $\phi \in G(R)$ so that $\phi(F) \subset E$ and so that $D(\phi(y), y) \in \Lambda$ for almost all $y \in F$. But by Proposition 8.4 of FMI (cf. also Definition 3.3.3 of Connes [1]), this is precisely the condition for $\lambda$ to be in $r^{*}(D)$.

3. Axiomatization of the $\mathbf{M}(R, \sigma)$. Proposition 2.9 identifies in $\mathbf{M}(R, \sigma)$, a particularly nice kind of abelian subalgebra which we call the diagonal subalgebra relative to the presentation of $\mathbf{M}$ as $\mathbf{M}(R, \sigma)$. We now axiomatize these properties as follows.

Definition 3.1. If $\mathbf{M}$ is a von Neumann algebra and $\mathbf{A}$ an abelian subalgebra, we shall say that $\mathbf{A}$ is a Cartan subalgebra if

(1) $\mathbf{A}$ is maximal abelian;

(2) $\mathbf{A}$ is regular;

(3) there exists a faithful normal conditional expectation of $\mathbf{M}$ onto $\mathbf{A}$.

After introducing this definition and proving the main theorem (Theorem 1 below) based on it, we discovered that Veršik [1] had introduced already the notion of a Cartan subalgebra. His definition is our (1) and (2), plus

there is a state $\omega$ of $\mathrm{A}$ such that $\omega(A B)=\omega(B A)$

for all $A \in \mathbf{A}$ and $B \in \mathbf{M}$.

It is not hard to see that this is the same condition.

Proposition 3.1. Condition (3) holds if and only if ( $\left.3^{\prime}\right)$ does. Moreover, the $E$ in (3) satisfies $E\left(U T U^{-1}\right)=U E(T) U^{-1}$ for $U \in \mathbf{N}(\mathbf{A})$.

Proof. If (3) holds, let $\omega$ be a faithful normal state on $\mathbf{A}$ and extend $\omega$ to a state (also denoted by $\omega$ ) on $\mathbf{M}$ by $\omega(T)=\omega(E(T)$ ). Then if $A \in \mathbf{A}$,

$$
\omega(A B)=\omega(E(A B))=\omega(A E(B))=\omega(E(B) A)=\omega(B A) .
$$

Conversely, if $\left(3^{\prime}\right)$ holds, let $\psi_{t}$ be the modular automorphism group associated to $\omega$. Since $\psi_{t}$ and $\omega$ satisfy the KMS condition it follows that $\psi_{t}(A)=A$ for all $A \in \mathrm{A}$. It follows by Takesaki [1] that there is a (unique) faithful normal conditional expectation $E$ of $\mathbf{M}$ onto $\mathbf{A}$ satisfying $\omega(E(T))=$ 
$\omega(T)$. The final statement of the proposition is in Theorem 1.5.5 of Connes [1].

Our main result provides as it were, a converse to the construction of $\$ 2$ and a structure theorem for certain von Neumann algebras.

THEOREM 1. Let $\mathbf{M}$ be a von Neumann algebra on a separable Hilbert space $\mathcal{H}$, and having a Cartan subalgebra $\mathbf{A}$. Then there exists a countable standard relation $R$ on a space $(X, \mathfrak{B}, \mu), \sigma \in H^{2}(R, \mathbf{T})$ and an isomorphism of $\mathbf{M}$ onto $\mathbf{M}(R, \sigma)$ carrying $\mathbf{A}$ onto the diagonal subalgebra of $\mathbf{M}(R, s)$. The relation $R$ is unique up to isomorphism, and the orbit of $\sigma$ under $N(R)$, the automorphism group of $R$, is unique.

Proof. The proof of this result is quite long and occupies the remainder of this section. It is arranged as a series of fifteen propositions, numbered 3.2 through 3.16. Our first remark is that we may take the central decomposition of $\mathbf{M}$ into factors $\mathbf{M}_{\boldsymbol{y}}$ which decomposes $\mathbf{A}$ as an integral of maximal abelian subalgebras $\mathbf{A}_{y}$ of $\mathbf{M}_{\text {y }}$. It is easy to verify that almost all $\mathbf{A}_{\boldsymbol{y}}$ are regular and that the conditional expectation $E$ decomposes as a direct integral of $E_{y}$ 's. It follows that if the theorem holds for factors so that $\mathbf{M}_{y} \simeq \mathbf{M}\left(R_{y}, \sigma_{y}\right)$ then a straightforward but tedious piecing together technique, using directly integral theory and appropriate measurable choice theorems, allows us to establish the theorem for general $\mathbf{M}$. Since our interest in the result is mainly for the case when $\mathbf{M}$ is a factor, we omit these details and assume forthwith that $\mathbf{M}$ is a factor. This effects some considerable notational simplification, and ease in the proof, but in fact our line of proof works in the general case.

For the initial part of the proof we shall assume weaker conditions on $\mathbf{A}$ than being a Cartan subalgebra. Namely, we assume (2) and (3) of Definition 3.1, but in place of (1) we assume that $\mathbf{A}$ is abelian and "normal" (see Fuglede-Kadison [1]), i.e., that $\mathbf{A}$ is the center of its centralizer in $\mathbf{M}$, or equivalently that $\mathbf{A}$ is the intersection of maximal abelian subalgebras of $\mathbf{M}$, or equivalently also that $\left(\mathbf{A} \vee \mathbf{M}^{\prime}\right) \cap \mathbf{M}=\mathbf{A}$. It is this last criterion that we shall use. It is also known that normality is also equivalent to the condition that the direct integral decomposition of the identity representation of a separable $C^{*}$ algebra $\mathbf{N}$ weakly dense in $\mathbf{M}$ with respect to $\mathbf{A}$ yields factor representations of $\mathbf{N}$ almost everywhere.

The part of the proof of Theorem 1 below, through Proposition 3.10, is valid with this weaker condition on $\mathbf{A}$, and we carry out the argument in this fashion.

Let $\mathbf{M} \supset \mathbf{A}$ be a factor with such an abelian subalgebra $\mathbf{A}$ and fix once and for all a faithful normal state $\omega$ on $A$. We identify $A=L^{\infty}(X)$ so that $\omega$ is represented by a probability measure on $X$. Then we extend $\omega$ to $\mathbf{M}$ by defining $\omega(T)=\omega(E(T))$ where $E$ is the given conditional expectation of $\mathbf{M}$ 
onto $\mathbf{A}$. Then by the properties of $E$, $\omega$ is a faithful normal state on $\mathbf{M}$. Then we may realize $M$ on a separable Hilbert space with a separating and cyclic vector $\phi_{0}$ with $\left(T \phi_{0}, \phi_{0}\right)=\omega(T), T \in M$. The Tomita-Takesaki theory produces an unbounded operator $\Delta$ on $\mathcal{H}$ so that $\Delta^{i t}$ induces a one parameter group of automorphisms of $\mathbf{M}$, and an anti-unitary $J$ such that $J \mathbf{M} J=\mathbf{M}^{\prime}$ is the commutant of $\mathbf{M}$.

We consider the abelian algebras $\mathbf{A}$ and $\mathbf{B}=J \mathbf{A} J$ which commute with each other and so generate an abelian von Neumann algebra $\mathbf{C}$. We have two *-isomorphisms $i$ and $j$ of $\mathrm{A}$ into $\mathrm{C}$, given by $i(A)=A$ and $j(A)=J A^{*} J$, and their images generate. Now we may realize $\mathbf{C}$ as $L^{\infty}(R, v)$ for some standard Borel space $R$ and measure (class) on $R$. Let us take $\nu(R)=1$. The corresponding $\tilde{i}$ and $\tilde{j}$ give (by point realization theorems) Borel maps $\pi_{l}$ and $\pi_{r}$ of $R$ into $X$ so that

$$
\tilde{i}(a)(z)=a\left(\pi_{l}(z)\right) \text { and } \tilde{j}(a)(z)=a\left(\pi_{r}(z)\right)
$$

for $\nu$ almost all $z \in R$. Since $i(A) \simeq \tilde{i}\left(L^{\infty}(X)\right)$ and $j(A) \simeq j\left(L^{\infty}(X)\right)$, and these generate $\mathrm{C} \cong L^{\infty}(R)$, it follows that the map $\pi(z)=\left(\pi_{l}(z), \pi_{r}(z)\right)$ of $R$ into $X \times X$ is almost everywhere injective since the algebras generated by the range of $\tilde{i}$ and $\tilde{j}$ respectively consist of functions constant on the fibers of the maps $\pi_{l}$ and $\pi_{r}$. Thus we may in effect assume that $R \subset X \times X$ and the $\pi_{l}, \pi_{r}$ are the projections to the first and second factors. We may assume that the measure $\nu$ projects under $\pi_{l}$ and $\pi_{r}$ to measures equivalent to the given measure $\mu$.

We also note that the map $A \mapsto J A^{*} J$ is a *-anti-automorphism of $\mathrm{B}(\mathcal{H C})$ which carries $\mathbf{A}$ onto $\mathbf{B}$ and $\mathbf{B}$ onto $\mathbf{A}$. It thus carries $\mathbf{C}$ onto itself and since $\mathbf{C}$ is abelian, it is a *automorphism of $\mathbf{C}$. It is therefore by general theory induced by a point map $\theta$ of $R$ onto itself, preserving the measure class of $\nu$. It is obvious, however, that in terms of the coordinates on $R$ as a subset of $X \times X, \theta(x, y)=(y, x)$. This shows that the flip $\theta$ on $X \times X$ carries $R$ onto itself a.e. and preserves the measure class of $\nu$. The outline of the argument is more or less clear at this point: we have to show that $R$ is a countable standard relation on $(X, \mathfrak{B}, \mu)$, that $\nu$ is equivalent to the counting measure, that the algebra $\mathrm{C}$ has uniform multiplicity one so that $\mathcal{H}=L^{2}(R, \nu)$ with $\mathrm{C}$ acting as multiplication operators, and finally, we have to produce a cocycle $s \in Z^{2}(R, \mathbf{T})$ so that $\mathbf{M}$ becomes naturally isomorphic to $\mathbf{M}(R, s)$.

Now the normalizer $\mathbf{N}(A)$ of $A$ in $M$ operates on $A$ as a group of automorphisms; moreover, $\mathbf{N}(\mathbf{A})$ acts on $\mathbf{B}=J A J \subset \mathbf{M}^{\prime}$ trivially so that $\mathbf{N}(\mathrm{A})$ acts as an automorphism of $\mathrm{C}$. Thus if $U \in \mathbf{N}(\mathrm{A}), U$ determines a Borel automorphism $\phi(U)$ of the space $X$ which leaves the measure $\mu$ quasiinvariant. The unitary $U$ also determines a Borel automorphism $\Phi(U)$ of $R \subset X \times X$ which by our remarks above is of the form $\Phi(U)(x, y)=(\phi(U)$ 
- $x, y)$. In a like manner any $V \in \mathbf{N}(\mathbf{B})$ normalizes $\mathbf{C}$ by operating trivially on A and so defines a Borel automorphism $\psi(V)$ of $X$ and one of $R$ of the form $\Psi(V)(x, y)=(x, \psi(V) y)$. Thus if an element $C$ of $\mathbf{C}$ is viewed as an element $c$ of $L^{\infty}(R, v)$,

$$
d(x, y)=c\left(\phi\left(U^{-1}\right)(x), \psi\left(V^{-1}\right)(x)\right),
$$

where $d$ corresponds to $(U V) C(U V)^{*}$.

Now let $G$ and $H$ be subgroups of $N(A)$ and $N(B)$ respectively, with $\mathbf{G} \supset \mathbf{U}(\mathbf{A}), \mathbf{H} \supset \mathbf{U}(\mathbf{B})$ so that $\mathbf{G} / \mathbf{U}(\mathbf{A})$ and $\mathbf{H}^{\prime} / \mathbf{U}(\mathbf{B})$ are countable and so that $G$ and $H$ are strongly dense in $\mathbf{N}(A)$ and $\mathbf{N}(B)$. It is possible to choose such groups, as $M$ is realized on a separable Hilbert space. Let $G=\mathbf{G} / \mathbf{U}(\mathbf{A})$ and $H=\mathbf{H} / \mathbf{U}(\mathbf{B})$ so that $G$ and $H$ are countable groups. Because of this countability, we may choose the point maps $\phi(U), U \in \mathbf{G}$, and $\psi(V), V \in \mathbf{H}$, so that up to null sets they depend only on the coset $\bmod U(A)$ of $U$ or mod $\mathbf{U}(\mathbf{B})$ of. $V$ respectively, and so that $\phi$ and $\psi$ define homomorphisms of $G$ and $H$ respectively into Borel automorphisms. Thus $G$ and $H$ act as countable Borel transformation groups on $X$, preserving the class of $\mu$, and $G \times H$ acts on $R$, preserving the class of $\nu$. We write the last action as $(g, h)(x, y)=$ $(g x, h y)$.

Proposition 3.2. The subalgebra of $\mathbf{C}$ of $G$ invariants is $\mathbf{B}$, and the subalgebra of $H$ invariants is $\mathbf{A}$. Hence the action of $G \times H$ is ergodic.

Proof. Since $A \cap \mathbf{B} \subset \mathbf{M} \cap \mathbf{M}^{\prime}$, which is the scalars, the last statement follows from the first two, and by symmetry, it suffices to consider the $G$ invariants. Let $C \in \mathrm{C}$ be fixed by $G$. Then by definition $U C U^{*}=C$ for all $U \in \mathbf{G}$. Then by continuity this is true for $U \in \mathbf{N}(\mathbf{A})$, but since the algebra generated by $\mathbf{N}(\mathbf{A})$ is weakly dense in $\mathbf{M}$ (A is regular), this says that $C$ commutes with $\mathbf{M}$, so in fact, $C \in \mathbf{M}^{\prime}$. But since $C \in \mathbf{C}$, which is generated by $\mathbf{A}$ and $\mathbf{B}, C$ is in the algebra $\mathbf{M} \vee \mathbf{B}$ generated by $\mathbf{M}$ and $\mathbf{B}$. But since $\mathbf{A}$ is normal in $\mathbf{M}, \mathbf{B}$ is likewise in $\mathbf{M}^{\prime}$, so by one of the criteria for normality, $(\mathbf{M} \vee \mathbf{B}) \cap \mathbf{M}^{\prime}=\mathbf{B}$ and it follows that $C \in \mathbf{B}$ as desired.

Proposition 3.3. The abelian algebra $\mathbf{C}$ has uniform multiplicity $m$ on $\mathcal{H}$, so that $\mathcal{H}$ may be realized as $L^{2}\left(R, \nu, \mathcal{H}_{0}\right)$, the space of square integrable $\mathcal{K}_{0}$ valued functions on $R$ with $\mathbf{C}$ acting as multiplication operators, where $\mathcal{F}_{0}$ is a fixed $m$ dimensional Hilbert space.

Proof. Let $R=\cup R_{n}$ be the partition of $R$ into subsets so that the characteristic function of $R_{n}$ is the projection $P_{n}$ onto the summand of multiplicity $n$. Then if $W \in \mathbf{N}(\mathbf{C}), W P_{n} W^{*}=P_{n}$ and in particular this is true for $W=U V$, with $U \in \mathbf{G}$ and $V \in \mathbf{H}$. It follows that $R_{n}$ is a $G \times H$ invariant set. By the proposition above, $R=R_{m}$ a.e. for some $m$. 
Now the measure $\nu$ on $R \subset X \times X$ can be disintegrated by the projections $\pi_{l}$ or $\pi_{r}$ to the first or second coordinates. The disintegration of $\nu$ with respect to $\pi_{r}$ gives base measure $\mu$ on $X$ and fiber measures $\beta_{y}$ on $X$, and similarly, disintegration with respect to $\pi_{r}$ yields $\mu$ as base measure on $X$ and fiber measures $\gamma_{x}$ on $X$, so that $\nu=\int_{X} \beta_{y} d \mu(y)$ and $\nu=\int_{X} \gamma_{x} d \mu(x)$. When we speak later about disintegration of a measure by a map, it is this construction to which we refer.

Proposition 3.4. Almost all of the fiber measures $\beta_{y}, y \in X$, (resp. $\gamma_{x}$, $x \in X)$ are quasi-invariant and ergodic under $G$ (resp. $H$ ).

Proof. It clearly suffices to consider the fiber measures $\beta_{y}$ on $X$. Since $\nu$ is quasi-invariant under the action of $G$ on $R \subset X \times X$ and since projection commutes with the action, the disintegration products $\beta_{y}$ are almost all quasi-invariant by Proposition 2.6 of Auslander-Moore [1]. If they were not almost all ergodic under $G$, there would be a set of positive measure $E \subset X$ and a nontrivial $H$-invariant set in the measure algebra $\Re\left(\beta_{y}\right)$ for $y \in E$. We now want to select, for each $y$ in a subset of positive measure in $E$, a $G$-invariant set $Z_{y} \subset X$ so that $\left\{(x, y): x \in Z_{y}\right\}$ defines a $\nu$-measurable subset of $R$. This is a tedious construction which we only outline. First we may pass to a subset of $E$ and assume that the measure algebras $\Re\left(\beta_{y}\right)$ are all isomorphic, say to $\Re(\beta)$ where $\beta$ is a fixed measure on $X$. Then we claim that we can find a function $\theta(x, y), y \in E$, so that for almost all $y \in E, \theta_{y}$ : $x \mapsto \theta(x, y)$ is a Borel isomorphism of $X$ onto $X$ so that $\left(\theta_{y}\right)_{*} \beta_{y} \sim \beta$ and so that $\theta(x, y)$ is jointly Borel in $x$ and $y$. We then form $\psi_{y}(g)(x)=\theta_{y}\left(g \theta_{y}^{-1}(x)\right)$ for $g \in G$, so that for almost all $y, g \mapsto \psi_{y}(g)$ defines an action of $G$ on $X$ which leaves $\beta$ quasi-invariant and so that $\psi_{y}(g)(x)$ is jointly Borel in $x$ and $y$ for each $g \in G$ (recall that $G$ is countable). Then we form the set $F=$ $\left\{(y, \alpha): \alpha \in \Re(\beta), \psi_{y}(g) \alpha=\alpha\right.$ for each $\left.g \in G\right\}$. It is not hard to verify that $F$ is a standard Borel space, and then by the measurable choice theorem (cf. Proposition 2.13 of Auslander-Moore [1]), we may find a function $f$ on $E$ so that $(y, f(y)) \in F$ for almost all $y \in E$, and we let $Z_{y}^{\prime}$ be a Borel set of class $f(y)$ with $\psi_{y}(g) Z_{y}^{\prime}=Z_{y}^{\prime}$. Then the choice $Z_{y}=\theta_{y}^{-1}\left(Z_{y}^{\prime}\right)$ provides the required choice. Then the set $\left\{(x, y): y \in E, x \in Z_{y}\right\}$ is $G$-invariant and measurable and as $G$ is countable, we can replace it by a $G$-invariant Borel set equal to it almost everywhere. The characteristic function of this set must be in $B \cong L^{\infty}(X)$ by Proposition 3.2, and this means that $Z_{y}$ has to be null or conull for almost all $y$, contrary to choice.

We now turn our attention to the separating and cyclic vector $\phi_{0}$ such that $\omega(T)=\left(T \phi_{0}, \phi_{0}\right)=\omega(E(T))=\left(E(T) \phi_{0}, \phi_{0}\right)$. Let $S=J \Delta^{1 / 2}$ be the polar decomposition of the conjugate linear map associated to $M$ and $\phi_{0}$ by $S\left(T\left(\phi_{0}\right)\right)$ $=T^{*}\left(\phi_{0}\right)$ for $T \in \mathbf{M}$. 
Proposition 3.5. For $A \in \mathbf{A},(J A J) \phi_{0}=A^{*}\left(\phi_{0}\right)$.

Proof. It is clear that $S\left(\phi_{0}\right)=\phi_{0}$ and $S^{*}\left(\phi_{0}\right)=\phi_{0}$, and so $J \phi_{0}=\phi_{0}$ and $\Delta^{1 / 2}\left(\phi_{0}\right)=\phi_{0}$. However, since $A$ is the centralizer of the state $\omega$ by condition (3') associated with Definition 3.1, it follows by the KMS condition that $\Delta^{i t}$ commutes with $\mathbf{A}$. Since $\phi_{0}$ and $A\left(\phi_{0}\right)$ for $A \in \mathbf{A}$ are in the domain of $\Delta^{1 / 2}$, $\Delta^{1 / 2} A \phi_{0}=A\left(\Delta^{1 / 2} \phi_{0}\right)=A \phi_{0}$. Thus $J A J\left(\phi_{0}\right)=J A\left(\phi_{0}\right)=J \Delta^{1 / 2} A\left(\phi_{0}\right)=$ $S\left(A \phi_{0}\right)=A^{*}\left(\phi_{0}\right)$ as desired.

But now if we realize $A \in \mathbf{A} \cong L^{\infty}(X)$ as multiplication by $a(x)$ on $\mathcal{H}=L^{2}\left(R, \nu, \mathcal{K}_{0}\right)$, then $J A^{*} J$ is realized (by the definition of $j$ above) as multiplication by $a(y)$. Now if $\phi_{0}$ is realized as an $L^{2}$ vector valued function $\phi_{0}(x, y)$, we find the following

COROLlaRY. $a(x) \phi_{0}(x, y)=a(y) \phi_{0}(x, y)$ for $\nu$ almost all $(x, y) \in R$, for each $a \in L^{\infty}(X)$.

Proposition 3.6. The function $\phi_{0}$ vanishes a.e. off the diagonal $\Delta(X) \subset X \times$ $X$, and in particular the diagonal $\Delta(X)$ has positive $\nu$ measure.

Proof. For any Borel subset $E$ of $X$, let $\psi(E)=(E \times E) \cup\left(E^{c} \times E^{c}\right)$. If $a=f_{E}$ is the characteristic function of $E, f_{E}(x) \phi_{0}(x, y)=f_{E}(y) \phi_{0}(x, y)$ a.e. If $(x, y) \in E \times E^{c}$, we obtain from the above $\phi_{0}(x, y)=0$ a.e., and similarly $\phi_{0}(x, y)=0$ a.e. on $E^{c} \times E$. Thus $\phi_{0}$ is supported on $\psi(E)$. Now let $E_{i}$ be a countable separating family of Borel subsets of $X$. Since $\phi_{0}=0$ a.e. outside $\psi\left(E_{i}\right), \phi_{0}=0$ outside $\bigcap_{i} \psi\left(E_{i}\right)$, which is just the diagonal $\Delta(X)$.

Proposition 3.7. The fiber measures $\beta_{y}$ of Proposition 3.4 give positive measure to the singleton set $\{y\}$ for $\mu$ almost all $y$. Similarly, $\gamma_{x}(\{x\})>0$ for almost all $x$.

Proof. It suffices by symmetry to consider the $\beta_{y}$. If $\beta_{y}(\{y\})=0$ for $y$ in a set $E$ of positive measure, let $A \in \mathbf{A}$ be the operator of multiplication by the characteristic function $a$ of the set $E$. Then $\left(a \phi_{0}\right)(x, y)=0$ unless $x=y \in E$; that is, unless $(x, y) \in \Delta(E)$. But $\nu(\Delta(E))=\int_{E} \beta_{y}(\{y\}) d \mu(y)$ by definition of disintegration, so $\Delta(E)$ is a null set, and $A \phi_{0}=0$. But $\phi_{0}$ is separating for $\mathbf{M} \supset \mathbf{A}$, and so this is impossible.

Proposition 3.8. For almost all $y, \beta_{y}$ is a purely atomic measure which gives positive mass to exactly the points of the $G$ orbit of $y$, and similarly for $\gamma_{x}$.

Proof. For almost all $y, \beta_{y}$ is quasi-invariant and ergodic. The atomic part of $\beta_{y}$ is an invariant set, so as $\beta_{y}$ has an atomic part, it is purely atomic. Quasi-invariance implies that $\beta_{y}(\{x\})>0$ iff $\beta_{y}(\{g \cdot x\})>0$ so $\beta_{y}$ gives positive mass to a union of orbits and by ergodicity it must be concentrated on one orbit, namely $G y$. 
Proposition 3.9. (1) If $\mathbf{G}$ and $\mathbf{G}_{1}$ are subgroups of $\mathbf{U}(\mathbf{A})$, both dense, and such that both $G=\mathbf{G} / \mathrm{U}(\mathrm{A})$ and $G_{1}=\mathrm{G}_{1} / \mathrm{U}(\mathrm{A})$ are countable, then the $G$ and $G_{1}$ orbits of almost all points are the same, so that the relations $R_{G}$ and $R_{G_{1}}$ coincide up to $\nu$-null sets. Furthermore, $\mu$ is quasi-invariant under $G$.

(2) We may take the set $R$ to be $R_{G}$ (or $R_{G_{1}}$ ) above and the measure $\nu$ is equivalent to a counting measure.

Proof. For (1) we note that the measure $\nu$ on $R$ is independent of any choice of $\mathbf{G}$, and Proposition 3.8 shows that $\nu$ determines $G y$ for almost all $y$, independently of $G$. It has already been noted that $\mu$ is quasi-invariant under G.

For (2) we note that Proposition 3.8 also says that the section $R(y)=\{x$ : $(x, y) \in R\}$ of $R$ at $y$ coincides with $G y$ up to $\beta_{y}$ sets of measure zero for $\mu$-almost all $y$. But this says that $R=R_{G}$ up to a set of $\nu$ measure zero so that we may take $R=R_{G}$. Since the disintegration of $\nu$ over projection to the second factor yields measures $\beta_{y}$ which give positive mass to precisely the points of the class $R(y)$ of $y$ with respect to $R$, it follows that $\nu$ is equivalent to right counting measure, since their common null sets $E \subset R$ consist of those $E$ such that $\{x:(x, y) \in E\}$ is void for $\mu$-almost all $y$.

Thus we have established our first major objective; that is, to show that $R$ can be taken to be a countable standard equivalence on $(X, \mathscr{B}, \mu)$ with $\nu$ equivalent to a counting measure. For convenience at this point, we replace finite $\nu$ with right counting measure on $R$, which we continue to denote by $\nu$. Then $\nu$ disintegrates over projection into the second factor with base measure $\mu$, and with fiber measures which we still denote by $\beta_{y}$, so that $\beta_{y}(F)=|F|$. The fiber measures $\gamma_{x}$ obtained by disintegrating with respect to projection to the first coordinate are of course expressed in terms of the Radon-Nikodym $D$ of $\mu$ with respect to the relation, specifically $\gamma_{x}(\{y\})=D(x, y)^{-1}=$ $D(y, x)$.

Now let $G(R)$ be the full group of the relation, the set of classes of Borel isomorphisms $\theta$ of $X$ to itself such that $\Gamma(\phi) \subset R$ with two being identified if they agree almost everywhere. Proposition 3.9 assures us that for any $U$ in $\mathbf{N}(\mathbf{A})$, the automorphism $A \mapsto U A U^{*}$ of $\mathbf{A}$ viewed as $L^{\infty}(X)$ is induced by a Borel isomorphism $\theta(U)$ sending $a$ to $a\left(\theta(U)^{-1} \cdot\right)$, with $\theta(U) \in N(R)$. In fact, $U \mapsto \theta(U)$ is a group homomorphism. Similarly, any $V \in \mathbf{N}(\mathbf{B})$ induces an automorphism $\rho(V)$ of $\mathbf{B}$ viewed as $L^{\infty}(X)$, sending $b$ to $b\left(\rho(V)^{-1} \cdot\right)$.

In view of the definition of the identification of $B$ with $A$, using the anti-unitary $J$, it follows that $\rho(J U J)=\theta(U)$ and so $\rho(V) \in G(R)$ also. The following allows us to analyze the structure of $\mathbf{N}(\mathbf{A})$ and $\mathbf{N}(\mathbf{B})$ acting on $\mathscr{H}=L^{2}\left(R, \nu, \mathcal{K}_{0}\right)$.

Proposition 3.10. For each $U \in \mathbf{N}(\mathbf{A})$, there exists a Borel map $a(U)$ from 
$R$ into the unitary group $\mathrm{U}\left(\mathcal{F}_{0}\right)$ of $\mathcal{F}_{0}$ so that $(U \psi)(x, y)=$ $a(U)(x, y) \psi\left(\theta(U)^{-1} x, y\right)$ for $\psi \in \mathcal{H}$. For each $V \in \mathbf{N}(\mathbf{B})$, there is a Borel map $b(V)$ from $R$ into $\mathrm{U}\left(\mathcal{F}_{0}\right)$ so that

$$
(V \psi)(x, y)=b(V)(x, y) \psi\left(x, \rho(V)^{-1} y\right) D\left(\rho(V)^{-1} y, y\right) .
$$

Proof. If $U \in \mathbf{N}(\mathrm{A})$, we define a unitary operator $U^{\prime}$ on $H$ by $\left(U^{\prime} \psi\right)(x, y)$ $=\psi\left(\theta(U)^{-1} \cdot x, y\right)$. Then $U^{\prime}$ evidently normalizes $\mathbf{C}$, the algebra generated by $\mathrm{A}$ and $\mathrm{B}$. Moreover, $U$ does also, and by definition, $U$ and $U^{\prime}$ induce the same automorphism of $\mathbf{C}$. Then $W=U U^{\prime-1}$ centralizes $\mathbf{C}$ and therefore has the form $(W \psi)(x, y)=a(U)(x, y) \psi(x, y)$ for a Borel function $a(U)$ from $R$ into $\mathbf{U}\left(\mathcal{F C}_{0}\right)$. Then $U=W U^{\prime}$ has the desired form.

If we are given $V \in \mathbf{N}(\mathbf{B})$, we define

$$
\left(V^{\prime} \psi\right)(x, y)=D\left(\rho(V)^{-1} y, y\right) \psi\left(x, \rho(V)^{-1} y\right)
$$

and verify that this is unitary. The argument proceeds exactly as in the first part.

The next step is to establish the basic fact that $\mathcal{K}_{0}$ is one dimensional: Here, of course, we must assume that $\mathbf{A}$ is a Cartan subalgebra rather than the weaker hypothesis we have been using up until now. Thus from now on, we assume in addition that $\mathbf{A}$ is maximal instead of being a normal abelian subalgebra.

Proposition 3.11. If $\mathbf{A}$ is a Cartan subalgebra, then $\mathcal{F}_{0}$ has dimension one.

Proof. Let $\phi_{0}$ be the separating and cyclic vector of Proposition 3.6, represented as a function $\phi_{0}(x, y)$ concentrated on the diagonal. Let $\phi_{0}(x)=$ $\phi_{0}(x, x)$; this is a unit vector since for every $A \in \mathrm{A}$, corresponding to $a \in L^{\infty}(X)$,

$$
\int a(x) d \mu(x)=\omega(A)=\left(A \phi_{0}, \phi_{0}\right)=\int a(x)\left|\phi_{0}(x)\right|^{2} d \mu(x)
$$

and it spans a one dimensional subspace $\mathcal{H}_{0}(x)$ of $\mathcal{H}_{0}$. By Proposition 3.10, for any $U \in \mathrm{N}(\mathrm{A}),\left(U \phi_{0}\right)(x, y)=0$ unless $x=\theta(U) y$ and then

$$
\left(U \phi_{0}\right)(\theta(U) y, y)=a(U)(\theta(U) y, y) \phi_{0}(y)
$$

for some unitary operator valued function as indicated.

Since $\mathbf{A}$ is regular, the linear combinations of the vectors $U\left(\phi_{0}\right), U \in \mathbf{N}(\mathbf{A})$, must be dense in $\mathcal{H}$. Now let $\mathcal{H}_{\Delta}$ be the subspace of functions in $\mathcal{H}$ supported on the diagonal and let $P_{\Delta}$ be the orthogonal projection onto $\mathcal{H}_{\Delta}$, which is, of course, just multiplication by the characteristic function of $\Delta$. Then $P_{\Delta}\left(U \phi_{0}\right)$ is a function which is zero off the diagonal, and at $(y, y)$ takes the value $I_{E}(y) a(U)(\theta(U) \cdot y, y) \phi_{0}(y)$ where $I_{E}$ is the characteristic function of a set $E \subset X$ and where $\theta(U) y=y$ for $y \in E$. 
Thus let us consider a $U \in \mathbf{N}(\mathbf{A})$ such that $\theta(U) y=y$ on a set $E$ of positive measure. Let $Q$ be the projection in $\mathbf{A}$ of the form $Q \psi(x, y)=$ $I_{E}(x) \psi(x, y)$ for $\psi \in \mathcal{H}$. It is clear from Proposition 3.10 that $U$ and $Q^{\prime}$ commute where $Q^{\prime}$ is any projection in $\mathrm{A}$ with $Q^{\prime}<Q$. Moreover, $U Q$ in fact commutes with $\mathrm{A}$. To see this it suffices to show that $U Q$ commutes with any projection in $\mathbf{A}$. But if $P \in \mathbf{A}, P=(1-Q) P+Q P$; we have already noted that $U Q$ commutes with $Q P$ as $Q P<Q$. Moreover, it is clear that $U Q$ times $(1-Q) P$ in either order is zero and so, in fact, $U Q$ commutes with $A$. But now $\mathbf{A}$ is maximal abelian so $Q U=U Q \in \mathbf{A}$ and therefore $Q U \psi(x, y)=$ $a(x) \psi(x, y)$ for some $a \in L^{\infty}(X)$. But on the other hand,

$$
Q U \psi(x, y)=I_{E}(x) a(U)\left(\theta(U)^{-1} x, y\right) \psi(x, y)=a(U)(x, y) \psi(x, y)
$$

for $x \in E$ and zero otherwise. By comparison we see that for $x \in E$, $a(U)(x, y)$ is, first of all, a scalar multiple of the identity and secondly is independent of $y$.

The first of these two facts tells us that $\left(P_{\Delta} U \phi_{0}\right)(x, x)=a(x) \phi_{0}(x)$ for some scalar valued function $a(x)$, and hence $\left(P_{\Delta} U \phi_{0}\right)(x, x) \in \mathcal{K}_{0}(x)$. But linear combinations of the $P_{\Delta}\left(U \phi_{0}\right)$ are dense in $\mathcal{H}_{\Delta}=L^{2}\left(\Delta, \mathcal{H}_{0}\right)$ and every such vector is of the form $a(x) \phi_{0}(x)$. The only way this can happen is for $\mathcal{H}_{0}=\mathcal{K}_{0}(x)$ to be of dimension one.

In the course of the argument we have established a fact that will be useful later and which we state separately.

Proposition 3.12. If $U \in \mathrm{N}(\mathrm{A})$ and $\theta(U) x=x$ for $x \in E$, then $a(U)(x, y)$ is independent of $y$ for $x \in E$, and $Q U=U Q$ is in $\mathbf{A}$ where $Q$ is the projection in A corresponding to $E$.

We have now achieved a substantial portion of our goal in that we now have $\mathcal{H}$ realized as $L^{2}(R, \nu)$ for a countable standard relation $R$ on $(X, \mathscr{B}, \mu)$ with $\mathbf{A}$ operating as multiplication operators in the first variable and $\mathbf{B}=$ $J A J$ as multiplication operators in the second variable. Moreover, operators in $\mathbf{N}(\mathbf{A})$ and $\mathbf{N}(B)$ have a form roughly of the right kind. What remains now is to refine the form of these operators and to produce a two cocycle $s$ so that $M$ is realized as $\mathbf{M}(R, s)$ on $\mathcal{H}=L^{2}(R, \nu)$. The separating cyclic vector $\phi_{0}$ can be realized as $\phi_{0}(x)$, a complex valued function of absolute value one. For simplicity we can adjust by a phase factor and assume that $\phi_{0}(x)=1$ so that $\phi_{0}$ is the characteristic function of the diagonal.

Now let us, as earlier, fix a group $G$ with $\mathbf{U}(\mathbf{A}) \subset \mathbf{G} \subset \mathbf{N}(\mathbf{A})$ with $\mathbf{G} / \mathbf{U}(\mathbf{A})$ countable and $\mathbf{G}$ dense in $\mathbf{N}(A)$. Let us select for each coset $g \in G=\mathbf{G} / \mathbf{U}(A)$ a representative $U(g)$ so that $U\left(g^{-1}\right)=U(g)^{-1}$ and $U(e)=1$. Then $G$ operates on $X$ so that $R=R_{G}$ and $g x=\theta(U(g)) x$, in the notation above. We let $\mathbf{D}$ be the set of all $U(g)$. Then if $(x, y) \in R$, there exists $U \in \mathbf{D}$ so 
that $\theta(U)^{-1} x=y$, although this $U$ may not be unique. In any case, the set of pairs $(x, y)$ with $\theta(U)^{-1} x=y$ is a Borel subset of $R$, and we may find a partition $\{A(U): U \in \mathbf{D}\}$ of $R$ into disjoint Borel sets so that $\theta(U)^{-1} x=y$ for $(x, y) \in A(U), A(1)=\Delta$, and $A\left(U^{-1}\right)$ is the flip of $A(U)$.

Proposition 3.13. (1) If we define $c(x, y, z)=a(U)(x, z)$ for $(x, y) \in$ $A(U)$, then $c$ is a well-defined Borel function on $R^{2}$ and satisfies $c(x, x, y)=1$, and $c(x, y, z)=c(y, x, z)^{-1}$ a.e.

(2) If $\left\{A^{\prime}(U): U \in \mathbf{D}^{\prime}\right\}$ is any partition of $R$, indexed by elements $U$ in a countable set $\mathbf{D}^{\prime} \subset \mathbf{N}(\mathbf{A})$ such that for $(x, y) \in A^{\prime}(U), \theta(U)^{-1} x=y$, and if $c^{\prime}(x, y, z)=a(U)(x, z)$ for $(x, y) \in A^{\prime}(U)$, then $c^{\prime}(x, y, z)=c(x, y, z) k(x, y)$ for some function $k$.

Proof. On the Borel set $A^{2}(U)=\{(x, y, z) ;(y, z) \in A(U)\} \subset$ $R^{2}, c(x, y, z)$ is a Borel function of the three variables. It follows that $c$ is a Borel function, as this is a countable partition. Since $a(I)(x, z)=1$ and $\Delta=A(1)$, it follows that $c(x, x, y)=1$. Moreover, it follows from Proposition 3.10 that $a\left(U^{-1}\right)(y, z)^{-1}=a(U)(\theta(U) g, z)$, and the second statement of (1) is an immediate consequence of this and the fact that $A\left(U^{-1}\right)$ is the flip of $A(U)$.

For (2), we consider the $A(U) \cap A^{\prime}(V)$. For $(x, y)$ in this set, $\theta(U)^{-1} x=y$ and $\theta(V)^{-1} x=y$ and so $\theta\left(U V^{-1}\right) x=x$, and it follows by Proposition 3.12 that $a\left(U V^{-1}\right)(x, z)$ is independent of $z$. If we expand this in terms of $a(U)$ and $a(V)$, we find that $a(U)(x, z) a(V)(x, z)^{-1}$ is independent of $z$. But this says that for $(x, y) \in A(U) \cap A^{\prime}(V), c(x, y, z) c(x, y, z)^{-1}$ is independent of $z$, and hence this holds globally for all $x, y$. If we call this function $k(x, y)$, then (2) follows.

Proposition 3.14. The function $c(x, y, r) c(y, z, r) c(x, z, r)^{-1}$ is for almost all $(x, y, z) \in R^{2}$ independent of $r$.

Proof. For each triple $(U, V, W)$ of elements of $\mathbf{D}$ let $E(U, V, W)=$ $\{(x, y, z):(x, y) \in A(U),(y, z) \in A(V)$ and $(x, z) \in A(W)\}$. Then the sets $E(U, V, W)$ form a partition of the set $R^{2}$. Moreover, it is evident that

$$
\theta(V)^{-1} \theta(U)^{-1}(x)=\theta(W)^{-1}(x)=z
$$

and so $\theta\left(U V W^{-1}\right) x=x$. Then by Proposition 3.12, $a\left(U V W^{-1}\right)(x, r)$ is independent of $r$ for almost all $x$ for which $(x, y, z) \in E(U, V, W)$. Again if we expand this, we find that it says that

$$
\begin{gathered}
a(U)(x, r) a(V)\left(\theta(U)^{-1} x, r\right) a(W)\left(\theta(W) \theta(U V)^{-1} x, r\right)^{-1} \\
=c(x, y, r) c(y, z, r) c(x, z, r)^{-1}
\end{gathered}
$$

is independent of $r$ for almost all $x$ such that $(x, y, z) \in E(U, V, W)$. 
We denote the function in the statement of the above proposition by $t(x, y, z)$. Note that if in place of $c$ we consider a function $c^{\prime}(x, y, z)=$ $c(x, y, z) k(x, y)$ as in (2) of Proposition 3.13, then the conclusion of 3.14 is still valid and we denote the resulting function by

$$
t^{\prime}(x, y, z)=c^{\prime}(x, y, r) c^{\prime}(y, z, r) c^{\prime}(x, z, r)^{-1} \text {. }
$$

Proposition 3.15. The function $t$ is a two cocycle, that is, it is in $Z^{2}(R, T)$. It is also a normalized cocycle, and moreover, its class $\sigma \in H^{2}(R, \mathbb{T})$ is independent of the choice of the $A(U)$ above, and hence depends only on $(\mathbf{M}, \mathbf{A})$ and the isomorphism of $\mathbf{A}$ with $L^{\infty}(X)$.

Proof. If the function $c$ itself were independent of its third variable, then the definition of $t$ would be simply $(\delta c)^{-1}$ and hence $\delta t=1$ as $\delta^{2}=1$. However, the same argument that shows that $\delta^{2}=1$ applies to $t$ as defined above and shows that $\delta(t)=1$, so that $t$ is a cocycle. Recall from the proof of Proposition 7.6 of FMI that a two-cocycle is normalized if it vanishes whenever any two of its variables are the same. The fact that $t(x, y, z)=1$ if $x=y$ or $y=z$ follows from the fact that $c(U, U, W)=1$ in Proposition 3.13 and the vanishing for $x=z$ follows from the fact that $c(U, V, W)=$ $c(V, U, W)^{-1}$, also in Proposition 3.13.

If we make different choices of the sets $A(U)$ and set $\mathbf{D} \subset \mathbf{N}(\mathbf{A})$ as described in (2) of Proposition 3.13, we obtain a new function $c^{\prime}(x, y, z)$ which differs from $c$ by a function $k(x, y)$ of the first two variables. Then evidently $t^{\prime}=s(\delta k)^{-1}$ is cohomologous to $t$.

Now let $k(x, y)=c(x, y, y)^{-1}$ and let $c^{\prime}(x, y, z)=c(x, y, z) k(x, y)$ so that $c^{\prime}(x, y, y)=1$ for almost all pairs $(x, y)$. Then if we form

$$
t^{\prime}(x, y, z)=c^{\prime}(x, y, r) c^{\prime}(y, z, r) c^{\prime}(x, z, r)
$$

which is independent of $r$ and is a cocycle equivalent to $t$, we may then for almost all triples $(x, y, z)$ evaluate this by taking $r=z$ in which case we see that $c^{\prime}(x, y, z)=t^{\prime}(x, y, z)$ is itself a cocycle, not necessarily normalized, however.

Now let $V \in \mathbf{N}(\mathrm{A})$, and let $E(U)=\left\{x:\left(x, \theta(v)^{-1} x\right) \in A(U)\right\}$. Then as $U$ runs over D, $E(U)$ is a partition of $X$, and $\theta(U)^{-1} x=\theta(V)^{-1} x$ for $x \in$ $E(U)$ so that $\theta\left(U V^{-1}\right) x=x$ for $x \in E(U)$. Then by Proposition 3.12, $a\left(U V^{-1}\right)(x, z)$ is independent of $z$ for $x \in E(U)$ and this means that we may write $a(V)(x, z)=a(U)(x, z) b_{U}(x)$ for $x \in E(U)$, where $b_{U}(x)$ is a function on $E(U)$ of absolute value one. If we let $b(x)=b_{U}(x)$ for $x \in E(U)$, then $a(V)(x, z)=c\left(x, \theta(V)^{-1} x, z\right) b(x)$ and we define

$$
\tilde{a}(V)(x, y)=b(x) k^{-1}\left(x, \theta_{1}(V)^{-1} \cdot x\right)
$$

for $y=\theta(V)^{-1} \cdot x$ and zero otherwise. Then 


$$
a(V)(x, z)=\tilde{a}(V)\left(x, \theta(V)^{-1} \cdot x\right) t^{\prime}\left(x, \theta(V)^{-1} \cdot x, z\right)
$$

so that

$$
(V \psi)(x, z)=a(V)(x, z) \psi\left(\theta(V)^{-1} \cdot x, z\right)=\sum_{y} \tilde{a}(V)(x, y) \psi(y, z) t^{\prime}(x, y, z) .
$$

Thus the operator $V$ has the proper form to be in one of our twisted matrix algebras. It will be helpful to bring this into the form of $\$ 2$ by replacing $t^{\prime}$ by a normalized cocycle equivalent to it. To do this we let $d$ be a function of absolute value one on $R$ which is one on the diagonal, and form the unitary operator $U_{d}$ of multiplication by $d$. Then instead of $\mathbf{M}$, we consider the algebra $U_{d} \mathbf{M} U_{d}^{-1}$ which contains $\mathbf{A}=U_{d} \mathbf{A} U_{d}^{-1}$ as a Cartan subalgebra. What this amounts to doing is modifying the identification of the Hilbert space $\mathcal{H}$ with $L^{2}(R, v)$ which we chose implicitly, immediately following Proposition 3.11. Since $d=1$ on $\Delta$, this does not change the representation of the distinguished separating and cyclic vector $\phi_{0}$ as the characteristic function of the diagonal. The calculation of Proposition 2.7 and the method of Proposition 7.7 of FMI for selecting a normalized cocycle equivalent to $t^{\prime}$ show that we may select $d$ so that $s=t^{\prime} \delta d$ is normalized and so that

$$
\left(U_{d} V U_{d}^{-1} \psi\right)(x, y)=\sum_{y}(d \tilde{a}(V))(x, y) \psi(y, z) s(x, y, z)
$$

for $V \in \mathbf{N}(\mathrm{A})$. (In fact, we could choose $d(x, y)$ to be a square root of $t^{\prime}(x, y, x)^{-1}$.) But now we may as well replace $\mathbf{M}$ by $U_{d} \mathbf{M} U_{d}^{-1}$ and having done so, we obtain the following:

Proposition 3.16. We may identify $\mathcal{H}$ with $L^{2}(R, v)$ and find a normalized cocycle $s$ in the cohomology class $\sigma$ of Proposition 3.15 so that any $V$ in the normalizer of $\mathbf{A}$ can be written as

$$
(V \psi)(x, z)=\sum_{y} \tilde{a}(V)(x, y) \psi(y, z) s(x, y, z)
$$

for a Borel function $\tilde{a}(v)$ which has absolute value one for $y=\theta(V)^{-1} \cdot x$ and vanishes otherwise.

Now let $\mathbf{M}_{0}$ be the algebra generated by $\mathbf{N}(\mathbf{A})$; that is, linear combinations of elements of $\mathbf{N}(\mathbf{A})$. Then by Proposition 2.4 it follows immediately that $\mathbf{M}_{\mathbf{0}}$ consists of precisely the operators of the form

$$
\left(L_{a} \psi\right)(x, z)=\sum_{y} a(x, y) \psi(y, z) s(x, y, z)
$$

where $a$ is a left finite function on $R$. Moreover, $\mathbf{M}_{0}$ is weakly dense in $\mathbf{M}$ by hypothesis. On the other hand, we have a von Neumann algebra $\operatorname{M}(R, s)$ by the construction of $\$ 2$ which contains a weakly dense subalgebra $\mathbf{L}$ (Definition 2.2) which is identical to $\mathbf{M}_{0}$. Thus $\mathbf{M}=\mathbf{M}(R, s)=\mathbf{M}(R, \sigma)$. Quite 
evidently, $R$ is determined by the pair $(\mathbf{M}, \mathbf{A})$ as it was constructed directly. The cohomology class $\sigma$ depended only on a fixed identification of $\mathbf{A}$ with $L^{\infty}(X)$. The ambiguity of $\sigma$ within its $N(R)$ orbit will be discussed in the next section on automorphisms.

4. Automorphisms. We have studied the structure of a pair (M, A) where $\mathbf{A}$ is a Cartan subalgebra, and now it is natural to turn to the question of automorphisms of such a pair. As usual, let Aut(M) be the full group of automorphisms of $\mathbf{M}$ and let $\operatorname{Out}(\mathbf{M})$, the outer automorphism group, be the quotient group of $\operatorname{Aut}(\mathbf{M})$ by the subgroup $\operatorname{Inn}(\mathbf{A})$ of inner automorphisms. Further, let Aut(M, A) be the group of automorphisms of $\mathbf{M}$ sending $\mathbf{A}$ into itself, and let $\operatorname{Out}(\mathbf{M}, \mathbf{A})$ be its image in Out(M). This can be described as those classes of outer automorphisms of $\mathbf{M}$ for which the image of $\mathbf{A}$ has the form $U A U^{-1}, U \in \mathbf{M}$. Recall that $G(R)$ denotes the full group of $R$; that is, (classes of) Borel automorphisms $\theta$ of $X$ with $\Gamma(\theta) \subset R$. Also, $N(R)$ denotes the normalizer of $R$; that is, (classes of) Borel automorphisms $\theta$ such that $\theta \times \theta(R) \subset R$. Finally, Out $(R)$, the outer automorphism group of $R$, is the quotient $N(R) / G(R)$.

If $H^{n}(R, A)$ is any cohomology group with a trivial $R$-module $A$, then $N(R)$ acts on $H^{n}(R, A)$ and in fact on the cocycle group $Z^{n}(R, A)$ by

$$
(\theta \cdot c)\left(x_{0}, \ldots, x_{n}\right)=c\left(\theta^{-1} \cdot x_{0}, \ldots, \theta^{-1} \cdot x_{n}\right)
$$

for $c \in Z^{n}(R, A)$. The action on cohomology results by passing to the quotient. Recall that we showed in Proposition 7.5 of FMI that if $\theta \in G(R)$, then $\theta \cdot c \sim c$ so that $G(R)$ operates trivially on $H^{n}(R, A)$. Now let $\sigma \in$ $H^{2}(R, \mathrm{~T})$ and let $N(R, \sigma)$ be the subgroup of $N(R)$ fixing $\sigma$ and let $\operatorname{Out}(R, \sigma) / G(R)$ be its image in $\operatorname{Out}(R)$. If $\theta \in N(R, \sigma)$ and if $s \in \sigma$ is a normalized cocycle, then there is a function $d(\theta)$ on $R^{2}$ with values in $T$ so that

$$
s\left(\theta^{-1}\left(x_{0}\right), \theta^{-1}\left(x_{1}\right), \theta^{-1}\left(x_{2}\right)\right)=(\delta d)^{-1}\left(x_{0}, x_{1}, x_{2}\right) s\left(x_{0}, x_{1}, x_{2}\right) .
$$

Since $\theta \cdot s$ and $s$ are both normalized it follows that $d(\theta)(x, x)=1$ and that $d(\theta)$ is skew symmetric. For simplicity, let $d$ (id) $(x, y)=1$. Finally, let $c \in$ $Z^{\prime}(R, \mathbf{T})$ and we define a map $\phi(c, \theta)$ on the set of all left finite functions by

$$
(\phi(c, \theta) a)(x, y)=c(x, y) d(\theta)(x, y) a\left(\theta^{-1}(x), \theta^{-1}(y)\right)
$$

Proposition 4.1. The map $L_{a} \mapsto L_{\phi(c, \theta) a}$ is $a^{*}$-automorphism of $\mathbf{L}$ which extends to $a *$-automorphism of $\mathbf{M}(R, s)$, also denoted by $\phi(c, \theta)$. It is spatially implemented by the unitary operator $U_{(c, \theta)}$ defined by

$$
\left(U_{(c, \theta)} \psi\right)(x, y)=c(x, y) d(\theta)(x, y) D\left(\theta^{-1}(y), y\right)^{1 / 2} \psi\left(\theta^{-1} x, \theta^{-1} y\right) .
$$


Proof. This is a straightforward calculation: note that the factor $D\left(\theta^{-1}(y), y\right)^{1 / 2}$ is necessary to make $U_{(c, \theta)}$ a unitary operator.

Now let $G(s)$ consist of all pairs $(c, \theta), c \in Z^{1}(R, \mathbf{T}), \theta \in N(R, \sigma)$. We define a multiplication on $G(s)$ by

$$
\left(c_{1}, \theta_{1}\right)\left(c_{2}, \theta_{2}\right)=\left(c_{1} \theta_{1}\left(c_{2}\right) d\left(\theta_{1}, \theta_{2}\right), \theta_{1} \theta_{2}\right)
$$

where $\theta_{1}\left(c_{2}\right)$ is the natural action of $\theta_{1}$ on $c_{2} \in Z^{1}(R, T)$ and where

$$
d\left(\theta_{1}, \theta_{2}\right)(x, y)=d\left(\theta_{1} \theta_{2}\right)(x, y) d\left(\theta_{1}\right)(x, y)^{-1} d\left(\theta_{2}\right)\left(\theta_{1}^{-1} \cdot x, \theta_{1}^{-1} \cdot y\right)^{-1} \text {. }
$$

The following extends results of Singer [1] and gives us the structure of $\operatorname{Aut}(\mathbf{M}, \mathbf{A})$ for $\mathbf{M}=\mathbf{M}(R, s)$.

THEOREM 2. The set $G(s)$ is a group and $(c, \theta) \mapsto \phi(c, \theta)$ is an isomorphism of $G(s)$ onto $\operatorname{Aut}(\mathbf{M}(R, s), \mathbf{A})$.

Proof. It is clear that $\phi(c, \theta)$ sends the diagonal algebra $\mathbf{A} \subset \mathbf{M}(R, s)$ onto itself and so $\phi(c, \theta) \in \operatorname{Aut}(\mathbf{M}(R, s), \mathbf{A})$. The product structure on $G(s)$ was concocted exactly so that $\phi$ is multiplicative, as a simple calculation verifies. Moreover, $\phi$ is injective, for if we apply $\phi(c, \theta)$ to an element $L_{a}$ of $A$, $a \in L^{\infty}(X)$, we obtain $L_{a^{\prime}}$ where $a^{\prime}(x)=a\left(\theta^{-1}(x)\right)$ and so if $\phi(c, \theta)=1$, it follows that $\theta=1$ and hence that $d(\theta)=1$ (by our choice). Then $(\phi(c, 1) a)(x, y)=c(x, y) a(x, y)$ for left finite $a$, and since we can find a countable number of left finite $a$ so that the sum of their absolute values is never zero, it follows that $c(x, y)=1$ a.e. Thus $\phi$ is injective and it follows that $G(s)$ is a group.

It remains to show that $\phi$ is surjective. Let $\alpha \in \operatorname{Aut}(M(R, s), \mathbf{A})$. Since $\mathbf{M}(R, s)$ has a separating and cyclic vector, the automorphism $\alpha$ is spatially induced by some unitary $U$ which by the arguments in Connes [2] we may take to commute with $J$. Note that the $U_{(c, \theta)}$ of Proposition 4.1 commutes with $J$. Now as $\alpha(A)=A, \alpha$ induces an automorphism $\theta$ of $L^{\infty}(X)$ so that $\alpha\left(L_{a}\right)=L_{a^{\prime}}$ where $a^{\prime}(x)=a\left(\theta^{-1} x\right)$. Now as $U \mathbf{M}(R, s) U^{-1}=\mathbf{M}(R, s)$, the same is true for the commutant; and as $U$ commutes with $J, U$ also normalizes $\mathbf{B}=J \mathbf{A} J$ and $\mathbf{C}$, the algebra generated by $\mathbf{A}$ and $\mathbf{B}$. Now for $b \in L^{\infty}(X)$,

$$
U R_{b} U^{-1}=U J L_{\bar{a}} J U^{-1}=J U L_{\bar{a}} U^{-1} J=J L_{\bar{a}^{\prime}} J=R_{b^{\prime}}
$$

where $a^{\prime}(x)=a\left(\theta^{-1} x\right)$. Finally, as $\mathbf{C} \approx L^{\infty}(R), U$ induces a Borel automorphism $\tilde{\theta}$ of $R$ so that $U M_{c} U^{-1}=M_{\tilde{c}}$ where $M_{\tilde{c}}$ is multiplication by $\tilde{c} \in$ $L^{\infty}(R)$ and $\tilde{c}(x, y)=\tilde{c}\left(\tilde{\theta}^{-1}(x, y)\right)$. But $\tilde{\theta}$ is determined completely by what happens on $\mathbf{A}$ and $\mathbf{B} \subset \mathbf{C}$; and it follows that $\tilde{\theta}$ has the form $\tilde{\theta}(x, y)=$ $(\theta(x), \theta(y))$. This shows that $\theta \times \theta(R) \subset R$ and hence $\theta \in N(R)$. Then we form the automorphism $\beta=\alpha \phi_{(1, \theta)}^{-1}$. If we can show that $\beta=\phi_{(c, 1)}$ for some $c \in Z^{1}(R, T)$, then $\alpha=\phi_{(c, \theta)}$, we are done. 
Now $\beta$ has the property that the induced automorphism of $\mathbf{A}$ is trivial. We may as before spatially implement $\beta$ by a unitary $V$ commuting with $J$. Now $V$ commutes with $\mathbf{A}$ and hence with $\mathbf{B}=J \mathbf{A} J$ and therefore with the algebra C they generate. But as $\mathrm{C}$ is maximal abelian, $V \in \mathrm{C}$, and so $V=M_{c}$ is multiplication by a function $c$ of absolute value one on $R$, which is skew symmetric as $V J=J V$. Then for any left finite $a, M_{c} L_{a} M_{c}^{-1}=L_{b}$ for some left finite $b$. When we write this out, it says that for any $a$ there is a $b$ so that $c(x, z) c(y, z)^{-1} a(x, y)=b(x, y)$ almost everywhere. Thus $a(x, y)(b(x, y))^{-1}$ $=d(x, y)$ is independent of $a$, and setting $y=z$, we see that $c(y, y) c(x, y)^{-1}$ $=d(x, y)$ a.e. so that $c(x, z) c(y, z)^{-1} c(x, y)^{-1}=c(y, y)$ a.e. Then if we put $x=z$, and use the skew symmetry of $c$, we see that $c(x, x)=c(y, y)$ is constant. Since $c$ is skew symmetric, this constant is either \pm 1 . If it is $+1, c$ is a cocycle. If it is -1 , it is clear that $-c$ is a cocycle and since $M_{c}$ induces the same automorphism $\beta$ as $M_{-c}$, we may replace $c$ by $-c$ in this case and assume that $c$ is a cocycle. Then $\beta=\phi_{(c, 1)}$.

THEOREM 3. The group $\operatorname{Aut}(\mathbf{M}(R, \sigma), \mathbf{A})$ is an extension of the normal abelian subgroup $Z^{1}(R, \mathbf{T})$ by $N(R, \sigma)$. If $\sigma=1$, the extension is a semidirect product.

Proof. The first statement is simply a rephrasing of Theorem 2. If $\sigma=1$, we may always take $d(\theta)=1$ and hence the factor $d\left(\theta_{1}, \theta_{2}\right)$ in the definition of $G(s)=G(1)$ may be taken to be 1 . Then evidently $G(1)$ is a semidirect product.

THEOREM 4. The group $\operatorname{Out}(\mathrm{M}(R, \sigma), \mathrm{A})$ is an extension of the abelian normal subgroup $H^{1}(R, \mathrm{~T})$ by $\operatorname{Out}(R, \sigma)$. If $\sigma=1$, the extension is the semidirect product.

Proof. The first statement is simply a matter of seeing when $\phi(c, \theta)$ is inner and given by some unitary $U \in \mathbf{M}(R, s)$. But then $U$ is in the normalizer N(A) of A. But by proof (3) of Proposition 2.9, we know that N(A) consists of operators of the form $L_{a(f, \phi)},|f|=1$ on $X, \phi \in G(R)$, and this unitary evidently induces the automorphism $\phi_{(\delta(\bar{\rho}, \phi)}$ so that $\phi_{(c, \theta)}$ is inner if and only if $c \in B^{1}(R, \mathbf{T})$ and $\theta \in G(R)$, and the first statement follows. The second statement is an immediate consequence of the second statement of Theorem 3.

It is appropriate at this point to treat the final item in the proof of Theorem 1 about the uniqueness of $\sigma$ in $\mathbf{M}(R, \sigma)$. We want to know that the pair $(\mathbf{M}, \mathbf{A})$ with $A$ a Cartan subalgebra determines $\sigma$ up to its orbit under $N(R)$ in $H^{2}(R, \mathbf{T})$. To see this consider $M_{1}=\mathbf{M}\left(R, s_{1}\right)$ with Cartan subalgebra $A_{1} \approx$ $L^{\infty}(X)$ and $\mathbf{M}_{2}=\mathbf{M}\left(R, s_{2}\right)$ with Cartan subalgebra $\mathrm{A}_{2} \approx L^{\infty}(X)$, and suppose that $\alpha$ is an isomorphism of $M_{1}$ onto $M_{2}$ which carries $A_{1}$ onto $A_{2}$. 
The isomorphism of $A_{1}$ onto $A_{2}$ determines an isomorphism $\beta$ of $L^{\infty}(X)$ onto $L^{\infty}(X)$, which is determined by a point map $\gamma \in N(R)$ so that $\beta(f)(x)=$ $f\left(\gamma^{-1} \cdot x\right)$. Now $\alpha$ must map $\mathbf{N}\left(\mathbf{A}_{1}\right)$ onto $\mathbf{N}\left(\mathbf{A}_{2}\right)$, and if $L_{a}^{i}$ is the operator in $\mathbf{M}_{i}$ with matrix given by the left finite function $a$, it is easy to see, as in the proof of Theorem 2, that $\alpha\left(L_{a}^{1}\right)=L_{\alpha(a)}^{2}$, where

$$
\alpha(a)(x, y)=d(x, y) a\left(\gamma^{-1}(x), \gamma^{-1}(y)\right)
$$

for some function $d$. But then $\alpha$ is multiplicative, and one sees that the only way this can happen is for $d(x, z) s_{1}\left(\gamma^{-1}(x), \gamma^{-1}(y), \gamma^{-1}(z)\right)$ to equal $d(x, y) d(y, z) s_{2}(x, y, z)$. This says, of course, that $s_{2}$ is equivalent to the transform of $s_{1}$ by $\gamma \in N(R)$. Thus if $\sigma_{i}$ is the class of $s_{i}, \sigma_{2}=\gamma \cdot \sigma_{1}$ so that the $N(R)$ orbit of $\sigma$ is uniquely determined.

We conclude with one other fact about automorphisms. Let $Z$ denote the centralizer of $\mathbf{A}$ in $\operatorname{Aut}(M), \mathbf{M}=\mathbf{M}(R, s)$; that is, the automorphisms fixing A elementwise. Then of course $Z \cong Z^{1}(R, T)$ and it is not hard to see that the usual topology on automorphisms of pointwise convergence in the predual of $\mathbf{M}$ coincides with the standard topology on $Z^{1}(R, \mathbf{T})$ in Moore [1] of convergence in measure. Now suppose that $A$ is an abelian locally compact group with dual group $\hat{A}$. Let $\gamma \in Z^{1}(R, \hat{A})$ and let $a \in A$ be viewed as a character of $\hat{A}$; that is, a group homomorphism of $\hat{A}$ into $\mathbf{T}$. Let $a(\gamma)$ be the image of $\gamma$ in $Z^{\prime}(R, \mathbf{T})$. One expects that $\phi_{\gamma}: a \mapsto a(\gamma)$ is a homomorphism of $A$ into $Z^{1}(R, T) \cong Z$; one is correct, and the converse is true:

THEOREM 5. The map $\phi_{y}$ is a continuous homomorphism of $A$ into $Z$; conversely every such continuous homomorphism is of the form $\phi_{\gamma}$ for a unique $\gamma \in Z^{1}(R, \hat{A})$.

Proof. The map $\phi_{\gamma}$ is clearly a homomorphism. If $a_{n} \rightarrow a$ in $A$, then $a_{n} \rightarrow a$ pointwise on $A$ and so $a_{n}(\gamma)(x, y)=a_{n}(\gamma(x, y))$ converges to $a(\gamma)(x, y)=$ $a(\gamma(x, y))$ and hence surely $a_{n}(\gamma) \rightarrow a(\gamma)$ in measure.

For the converse consider the polonais group $U(A, \mathbf{T})$ of classes of Borel functions from $A$ (identified via Haar measure on $A$ ) into T. Then $\hat{A}$ viewed as characters on $A$ is injected into $U(A, \mathbf{T})$. It is a consequence of standard facts in Fourier analysis that the image is closed: it consists of those $f$ which are homomorphisms almost everywhere, i.e., $f\left(a_{1} a_{2}\right)=f\left(a_{1}\right) f\left(a_{2}\right)$ for almost all pairs. Then $U(R, \hat{A})$ is injected into $U(R, U(A, \mathbf{T}))$ and the subgroup $Z^{\prime}(R, \hat{A})$ is injected into $Z^{\prime}(R, U(A, \mathbf{T}))$; by the above, the range may be characterized as those $s$ such that $s(x, y)$ is for almost all $(x, y) \in R$ a homomorphism almost everywhere. But now by the Fubini theorem in Moore [1], $U(R, U(A, \mathbf{T}))$ can be identified with $U(A, U(R, \mathbf{T}))$, and a moment's reflection shows that the subgroup $Z^{1}(R, U(A, \mathrm{~T}))$ is then identified with the subgroup $U\left(A, Z^{1}(R, \mathbf{T})\right)$ of functions on $A$ taking values almost everywhere 
in $Z^{1}(R, \mathbf{T})$. But now the generalized direct integral theorem, Theorem 1 of Moore [4] says that the set of $s \in Z^{1}(R, U(A, \mathbf{T}))$ so that $s(x, y)$ is a homomorphism, i.e., $s(x, y) \in \hat{A}$ viewed as a subgroup of $U(A, \mathbf{T})$, corresponds under this identification to the elements in $U\left(A, Z^{1}(R, \mathbb{T})\right)$ which are homomorphisms.

All of this says that the natural map of $Z^{1}(R, \hat{A})$ into $U\left(A, Z^{1}(R, \mathbf{T})\right)$ by $\gamma \mapsto \phi_{\gamma}$ where $\phi_{\gamma}(a)=a(\gamma)$, is injective, with image equal to the (continuous) homomorphisms from $A$ to $Z^{1}(R, \mathbf{T})$.

As a special case of this, we see that the one parameter groups in $Z=Z^{1}(R, \mathbf{T})$ are indexed by the elements of $Z^{1}(R, \mathbf{R})$. If $D$ is the RadonNikodym cocycle for instance, then $\log (D) \in Z^{1}(R, \mathbf{R})$ and the corresponding one parameter group of automorphisms is the modular group of $M$ associated to the state $\omega$. In this connection it is timely now to give the proof alluded to in Remark 2 after Theorem 8 of FMI. More precisely, we prove the following fact:

THEOREM 6. If $c \in Z^{1}(R, \mathbf{R})$ and is essentially bounded, then it is trivial.

Proof. By the above, $\exp (i t c(x, y))$ defines a one parameter group $\phi_{t}$ of automorphisms in $Z$. However, $\phi_{t}$ is evidently norm continuous as the infinitesimal generator $M_{c}$ is bounded. Then by Kadison-Ringrose [1], $\phi_{t}$ is inner, so that $\phi_{t}(W)=U_{t} W U_{t}^{*}$ for a one parameter unitary group in $\mathbf{M}$. But $U_{t}$ is in the centralizer of $\mathbf{A}$, as $\phi_{t} \in Z$ and so $U_{t}$ is in $\mathbf{A}$. Thus there exists a real valued Borel function $d$ on $X$ so that $U_{t}=L_{\text {exp }(i t) \text {. }}$. By previous calculations, this says that $\phi_{t}$ is also determined by the one-cocycle $\exp (i t(d(x)-$ $d(y)))$. By injectivity in Theorem 2, $\exp (i t(d(x)-d(y)))=\exp (i t c(x, y))$ almost everywhere for each $t$. By a standard argument we deduce that $c(x, y)=d(x)-d(y)$ almost everywhere.

Theorem 8 of Greenleaf [1] states that if $G$ is locally compact separable and amenable, but not necessarily discrete, and acts on a Borel space $X$ leaving a measure $\mu$ quasi-invariant and has a uniform bound for the Radon-Nikodym derivatives, $(d g \cdot \mu / d \mu)$, then there is an equivalent invariant measure. We stated that in a subsequent paper the result would be established in general without the hypothesis of amenability. The result above does just this for $G$ discrete. For general $G$, one could form the continuous analogue of our construction of $\mathrm{M}(R, 1)$ using $R=R_{G}$. We could then apply the same argument using the Kadison-Ringrose result. The technique of course proves that any bounded cocycle is trivial.

5. Different Cartan subalgebras. We have studied the structure of a pair (M, A) with A a Cartan subalgebra and the automorphisms of such a pair. We now turn to the question of different Cartan subalgebras in a given $\mathbf{M}$ and conjugacy questions. First, a piece of terminology; a Cartan subalgebra $\mathbf{A}$ 
of $\mathbf{M}$ will be called hyperfinite if the resulting relation $R$ which is determined by the pair is hyperfinite. In that case, $H^{2}(R, \mathbf{T})=0$ by Theorem 6 of FMI, so $\mathbf{M}=\mathbf{M}(R, 1)=\mathbf{M}(R)$ is determined by $R$. If $R$ is also ergodic, so that $\mathbf{M}$ is a factor, $\mathbf{M}$ is called a Krieger algebra or a Krieger factor.

Now if $\mathbf{A}_{1}$ and $\mathbf{A}_{2}$ are two Cartan subalgebras of a von Neumann algebra $\mathbf{M}$, one says that $\mathbf{A}_{1}$ and $\mathbf{A}_{2}$ are inner conjugate if there is a unitary $U$ in $\mathbf{M}$ with $U A_{1} U^{-1}=A_{2}$; one says that they are conjugate, or (if necessary to be more precise), outer conjugate, if there is an automorphism $\phi$ of $\mathbf{M}$ with $\phi\left(\mathbf{A}_{1}\right)=\mathbf{A}_{2}$.

It is of course too much to ask that all Cartan subalgebras be inner conjugate. In fact, we point out the following fact, which is undoubtably known to others.

THEOREM 7. The hyperfinite $\mathrm{II}_{1}$ factor $\mathbf{M}$ has two hyperfinite Cartan subalgebras which are conjugate but not inner conjugate.

Proof. According to Connes [4], Out(M) is a simple group. Let $\mathbf{A}$ be a hyperfinite Cartan subalgebra, so that $\mathbf{M}=\mathbf{M}(R)$ where $R$ is the unique ergodic hyperfinite relation of type $\mathrm{II}_{1}$. If every outer conjugate of $\mathbf{A}$ were also inner conjugate to $A$, then in the language of Theorem $4, \operatorname{Out}(\mathbf{M}, \mathbf{A})=$ Out(M) is an extension of a normal abelian group $H^{1}(R, T)$ by another group $\operatorname{Out}(R)$. But both of these groups are maifestly nonzero so Out(M) is not simple.

One does have one very beautiful positive fact due to Krieger [2], which in the present language becomes the following statement:

TheOREM 8 (KRIEGer). Any two hyperfinite Cartan subalgebras of a von Neumann algebra $\mathbf{M}$ are conjugate.

Proof. The situation can be immediately reduced to the case of a factor by direct integral theory. If $A_{1}$ and $A_{2}$ are two such algebras, then $\mathbf{M} \simeq \mathbf{M}\left(R_{1}\right) \simeq$ $\mathbf{M}\left(R_{2}\right)$ for two hyperfinite ergodic relations. Krieger's result is then that $R_{1}$ is isomorphic to $R_{2}$ and this is just another way of saying that $\mathbf{A}_{1}$ and $\mathbf{A}_{2}$ are conjugate.

Other than the above, the state of knowledge is rather abysmal. For instance, we do not know if an algebra $\mathbf{M}$ could have Cartan subalgebras $\mathbf{A}_{1}$ and $\mathbf{A}_{2}$ with $\mathbf{A}_{1}$ hyperfinite but $\mathbf{A}_{2}$ not hyperfinite. There is one further example of some interest. Connes [3] produces an algebra not anti-isomorphic to itself, or equivalently not isomorphic to its opposed algebra. This example is of the form $\mathbf{M}(R, \sigma)$ and since the opposed algebra of $\mathbf{M}(R, \sigma)$ is evidently $\mathbf{M}\left(R, \sigma^{-1}\right)$, we see that Connes' example cannot have any Cartan subalgebra B for which $\mathbf{M}=M\left(R^{\prime}, 1\right)$ for the relation $R^{\prime}$ associated to $\mathbf{B}$ and the trivial cohomology class 1 . 
The remainder of this section is devoted to a study of inner conjugacy of Cartan subalgebras. The main result gives three equivalent conditions for this to happen at least for algebras of purely infinite type. The situation is analogous to our comparison in Theorem 3 of FMI between isomorphism of relations and the weaker condition that they define the same virtual group. We make the following definition in which $\mathbf{B}(\mathcal{H C})$ is the algebra of all bounded operators on an infinite dimensional Hilbert space, and $A(\mathcal{H C})$ is the subalgebra of infinite diagonal matrices, i.e., a Cartan subalgebra of $\mathbf{B}(\mathcal{H C})$.

Definition 5.1. Let $M$ be a von Neumann algebra and $A_{1}$ and $A_{2}$ two maximal abelian subalgebras. We say that $A_{1}$ and $A_{2}$ are (inner) coconjugate if there is an (inner) automorphism $\phi$ of $\mathbf{M} \otimes \mathbf{B}(\mathcal{H C})$ which carries $\mathbf{A}_{1} \otimes \mathbf{A}(\mathcal{H})$ onto $A_{2} \otimes A(\mathcal{H C})$.

We first show that this implies conjugacy if $\mathbf{M}$ is purely infinite.

Proposition 5.1. If $M$ is purely infinite then two subalgebras $\mathbf{N}_{1}$ and $\mathbf{N}_{2}$ are (inner) coconjugate if and only if they are (inner) conjugate.

Proof. Let $\mathbf{M}$ be purely infinite and let $\mathbf{A}$ be maximal abelian. We can then find an infinite sequence of orthogonal projections $P_{i}$ in A with sum $I$ and isometries $U_{i} \in \mathbf{M}$ with initial projection $I$ and terminal projection $P_{i}$. If $M \in \mathbf{M}$, we let $M_{i j}=U_{i}^{*} M U_{j}$ where each $M_{i j} \in \mathbf{M}$, and we view the matrix $\left(M_{i j}\right)$ as an element of $\mathbf{M} \otimes \mathbf{B}(\mathcal{H C})$. It is elementary that $\phi: M \rightarrow\left(M_{i j}\right)$ is an isomorphism of $\mathbf{M}$ onto $\mathbf{M} \otimes \mathbf{B}(\mathcal{H})$. Moreover, since $P_{i} \in \mathbf{A}$ and $\mathbf{A}$ is abelian it is clear that $\phi(\mathbf{A})=\mathbf{A} \otimes \mathbf{A}(\mathcal{H})$.

Now if we are given $A_{1}$ and $A_{2}$ we construct maps $\phi_{1}$ and $\phi_{2}$ of the kind described above from $\mathbf{M}$ onto $\mathbf{M} \otimes \mathbf{B}(\mathcal{T C})$. Then if $\theta$ is an automorphism of $\mathbf{M} \otimes \mathbf{B}(\mathcal{H C})$ which sends $\mathbf{A}_{1} \otimes \mathbf{A}(\mathcal{F C})$ onto $\mathbf{A}_{2} \otimes \mathbf{A}(\mathcal{H C})$, then $\psi=\phi_{2}^{-1} \theta \phi_{1}$ is an automorphism of $\mathbf{M}$ carrying $\mathbf{A}_{1}$ onto $\mathbf{A}_{2}$. This shows that if $\mathbf{A}_{1}$ and $\mathbf{A}_{2}$ are coconjugate, then they are conjugate. If $\mathbf{A}_{1}$ and $\mathbf{A}_{2}$ are inner conjugate so that we may take $\theta$ inner and given by a unitary $W$ with a matrix $\left(W_{i j}\right)$ with $W_{i j} \in \mathbf{M}$, then one can easily construct a unitary in $\mathbf{M}$ which implements $\psi$, namely $\Sigma_{i l} V_{i} W_{i l} U_{l}^{*}$ where $U_{i}$ are the partial isometries used to define $\phi_{1}$ and $V_{j}$ are the partial isometries used to define $\phi_{2}$.

In order to state our theorem we must introduce one more concept. Let $\mathbf{A}$ and $\mathbf{B}$ be abelian von Neumann algebras and let $F$ be a positive normal map from $\mathbf{B}$ to $\mathbf{A}$ which takes $I$ to $I$. Now, let $\mathbf{A} \cong L^{\infty}(X), \mathbf{B} \cong L^{\infty}(Y)$, and let $\mu$ be a probability measure on $X$ defining $L^{\infty}(X)$. The following structure theorem for such maps $F$ must be known and we omit the simple proof.

Proposition 5.2. Given $F$, there exists a unique probability measure $\tau$ on $X \times Y$ with disintegration products $\tau_{x}$ with respect to projection to $X$, which are probability measures on $Y$ so that $F(f)(x)=\int f(y) d \tau_{x}(y)$ for almost all $x$. Conversely, every such $\tau$ determines an $F$. 
Definition 5.2. Let $F$ be as above; we say that $F$ is discrete if $\tau_{x}$ is an atomic measure for almost all $x$.

Definition 5.3. Let $\mathbf{M}$ be a von Neumann algebra with Cartan subalgebra $\mathbf{A}$ and let $\mathbf{B}$ be an abelian subalgebra. We say that $\mathbf{B}$ is discrete over $\mathbf{A}$ if the restriction to $\mathbf{B}$ of the conditional expectation $E$ of $\mathbf{M}$ onto $\mathbf{A}$ is discrete. (We have noted earlier that $E$ is uniquely determined by $\mathbf{A}$ so that this depends only on A.)

THEOREM 9. Let $\mathbf{A}_{1}$ and $\mathbf{A}_{2}$ be Cartan subalgebras of the von Neumann algebra $\mathbf{M}$. Then $\mathbf{A}_{1}$ and $\mathbf{A}_{2}$ are inner coconjugate iff $\mathbf{A}_{1}$ is discrete over $\mathbf{A}_{2}$ and $\mathbf{A}_{2}$ is discrete over $\mathbf{A}_{1}$.

Proof. We reduce to case of a factor by direct integral decompositions. We observe also that $\mathbf{A}_{1}$ is discrete over $\mathbf{A}_{2}$ iff $\mathbf{A}_{1} \otimes \mathbf{A}(\mathcal{C})$ is discrete over $\mathbf{A}_{2} \otimes \mathbf{A}(\mathcal{H})$ since the positive map of $\mathbf{A}_{1} \otimes \mathbf{A}(\mathcal{H})$ into $\mathbf{A}_{2} \otimes \mathbf{A}(\mathcal{H})$ is just $E \otimes I$ where $E$ is the positive map of $A_{1}$ into $A_{2}$. Thus we may assume that $\mathbf{M}$ is an infinite factor and then by Proposition 5.1, our assertion is that $\mathbf{A}_{1}$ and $\mathbf{A}_{2}$ are inner conjugate iff the discreteness condition holds.

First suppose that $\mathbf{A}_{2}=U \mathbf{A}_{1} U^{*}$ and by Theorem 1 , write $\mathbf{M}=\mathbf{M}(R, s)$ with $A_{1}$ as diagonal subalgebra. Then the unitary operator $U$ is of the form $L_{u}$ for a function $u$ on $R$. Then if $A \in \mathrm{A}_{1}$ is represented by $a \in L^{\infty}(X)$, the operator $U A U^{-1}=L_{b}$ where

$$
b(x, z)=\sum_{y} a(y)(u(x, y) \bar{u}(z, y) s(x, y, z)) .
$$

Then the conditional expectation of $L_{b}$ is the function $b(z, z) \in L^{\infty}(X)$. Thus $\mathbf{A}_{1} \cong L^{\infty}(X)$; and if we identify $\mathbf{A}_{2}$ with $L^{\infty}(X)$ via conjugation by $U$, the positive map from $\mathbf{A}_{2}$ to $\mathbf{A}_{1}$ can be viewed as the map $F$ from $L^{\infty}(X)$ to $L^{\infty}(X)$ given by

$$
(F(a))(z)=\sum_{x} a(x)|u(z, x)|^{2}
$$

Thus the disintegration products $\tau_{x}$ of the measure $\tau$ of Definition 5.1 are atomic measures giving mass $|u(z, x)|^{2}$ to all points $z$ in the equivalence class of $x$. Thus $\mathbf{A}_{2}$ is discrete over $\mathbf{A}_{1}$ and by a symmetric argument, $\mathbf{A}_{1}$ is discrete over $\mathbf{A}_{2}$.

Now suppose that the discreteness condition is satisfied, and let $\mathbf{A}_{i}=$ $L^{\infty}\left(X_{i}, \mu_{i}\right)$ with $\mu_{i}$ probability measures on $X_{i}$. We realize $\mathbf{M}$ as $\mathbf{M}\left(R_{1}, s_{1}\right)$ for a relation $R_{1}$ on $X_{1}$ and let $J_{1}$ be the conjugate linear " $J$ " arising from this construction. Let $\mathbf{B}_{1}=J_{1} \mathbf{A}_{2} J_{1}$, which is a Cartan subalgebra of $\mathbf{M}^{\prime}$ and isomorphic to $\mathbf{A}_{1}$ and $L^{\infty}\left(X_{1}\right)$. Now we let $\mathbf{D}$ be the abelian algebra generated by $\mathbf{B}_{1}$ and $A_{2}$. As in the proof of Theorem 1 , we may identify $\mathbf{D}$ with $L^{\infty}(S, \lambda)$ where $S \subset X_{1} \times X_{2}$ with $\mathbf{B}_{1}$ consisting of functions constant in the right 
coordinate and $A_{2}$ of functions constant in the left coordinate, and where $\lambda$ is some measure in $S$ projecting to $\mu_{i}$ on $X_{i}$. Now, as $A_{1}$ is also isomorphic to $L^{\infty}\left(X_{1}\right)$, restriction to $\mathbf{A}_{2}$ of the conditional expectation $E_{1}$ of $\mathbf{M}$ onto $\mathbf{A}_{1}$ gives a positive map $F$ from $L^{\infty}\left(X_{2}\right)$ to $L^{\infty}\left(X_{1}\right)$ and hence by Proposition 5.2 we have a measure $\tau$ on $X_{1} \times X_{2}$ and disintegration products $\tau_{x}, x \in X_{1}$. The first step is to connect $\tau$ and $\lambda$.

Proposition 5.3. The measure $\tau$ is absolutely continuous with respect to $\lambda$.

Proof. Let $\phi_{1}$ be the separating and cyclic vector for $\mathbf{M}$ associated with its presentation as $\mathbf{M}\left(K_{1}, s_{1}\right)$, and $\omega_{1}$ the corresponding state. If $d \in L^{\infty}(S)$ and $M_{d}$ is the corresponding operator in $\mathbf{D}$, then the map $d \mapsto\left(M_{d} \phi_{1}, \phi_{1}\right)$ defines a measure $\rho$ on $S$, absolutely continuous with respect to $\lambda$. We claim, in fact, that $\rho=\tau$. To see this it suffices to consider functions $d$ of the form $b\left(x_{1}\right) a\left(x_{2}\right)$ which represent operators $R_{b}=M_{b} \in \mathrm{B}_{1}$ and $M_{a} \in \mathrm{A}_{2}$. Then

$$
\int b\left(x_{1}\right) a\left(x_{2}\right) d \rho=\left(M_{a} J_{1} L_{b} J_{1} \phi_{1}, \phi_{1}\right)=\left(M_{a} L_{b} \phi_{1}, \phi_{1}\right)
$$

since $J_{1} \phi_{1}=\phi_{1}$ and since $J_{1} L_{b} \phi_{1}=L_{b} \phi_{1}$. Then

$$
\begin{aligned}
\left(M_{a} L_{b} \phi_{1}, \phi_{1}\right) & =\omega\left(M_{a} L_{b}\right)=\omega\left(E\left(M_{a} L_{b}\right)\right)=\omega\left(E\left(M_{a}\right) L_{b}\right) \\
& =\int\left(\int a\left(x_{2}\right) d \tau_{x_{1}}\right) b\left(x_{1}\right) d \mu_{1}\left(x_{1}\right) \\
& =\int a\left(x_{2}\right) b\left(x_{1}\right) d \tau\left(x_{1}, x_{2}\right)
\end{aligned}
$$

by definition of $\tau_{x_{1}}$ and $\tau$.

Now we can proceed to repeat the arguments given in Propositions 3.2, 3.3 and 3.4 in the course of the proof of Theorem 1, but applied to the measure $\lambda$ on $S \subset X_{1} \times X_{2}$. We produce countable groups $H_{1}$ and $G_{2}$ of Borel automorphisms of $X_{1}$ and $X_{2}$ from the normalizers $\mathbf{N}\left(\mathbf{B}_{1}\right)$ and $\mathbf{N}\left(\mathbf{A}_{2}\right)$ respectively so that, in fact, $H_{1}$ generates the relation $R_{1}$ on $X_{1}$, and $G_{2}$ generates the relation $R_{2}$ on $X_{2}$ (with $\mathbf{M}=M\left(\mathbf{R}_{2}, s_{2}\right)$ ), and so that when we disintegrate $\lambda$ with respect to projection to $X_{1}$ we have fiber measures $\lambda_{x}\left(x \in X_{1}\right)$ which are all ergodic for $G_{2}$ and similarly for projection to the right coordinate.

But now $\tau<\lambda$ so that $\tau_{x}<\lambda_{x}$ for almost all $x$. Since $\tau_{x}$ is atomic, it follows from ergodicity of $\lambda_{x}$ that it is also atomic, and indeed is equivalent to a counting measure on a certain $G_{2}$ orbit or, equivalently, an $R_{2}$ equivalence class in $X_{2}$.

PROPOSITION 5.4. For almost all $x, \lambda_{x}$ is equivalent to a counting measure on an $R_{2}$ class in $X_{2}$ and $\lambda_{x} \sim \lambda_{x^{\prime}}$ when $\left(x, x^{\prime}\right) \in R_{1}$. Similarly, if $\lambda^{y}, y \in R$, are the disintegration products of $\lambda$ with respect to projection to the second factor, then $\lambda^{y}$ is equivalent to a counting measure on an $R_{1}$ equivalent class in $X_{1}$ and $\lambda^{y} \sim \lambda^{y^{\prime}}$ if $\left(y, y^{\prime}\right) \in R_{2}$. 
The statements about $\lambda^{y}$ follow in exactly the same way by making use of the hypothesis that $\mathbf{A}_{1}$ is discrete over $\mathbf{A}_{2}$.

The set $S \subset X_{1} \times X_{2}$ has the property that measure theoretically any section $S_{1}\left(x_{1}\right)$ at a point $x_{1} \in X_{1}$ is countable and is an equivalence class of $R_{2}$, and that any horizontal section $S_{2}\left(x_{2}\right)$ at $x_{2} \in X_{2}$ is countable and an equivalence class of $R_{1}$.

Proposition 5.5. The set $S$ contains the graph of a map from $X_{1}$ to $X_{2}$ which is an equivalence of $R_{1}$ with $R_{2}$.

Proof. By standard cross section theorems we may find maps $\phi_{1}$ from $X_{1}$ to $X_{2}$ and $\phi_{2}$ from $X_{2}$ to $X_{1}$ so that $\left(x_{1}, \phi_{1}\left(x_{1}\right)\right) \in S$ and $\left(\phi_{2}\left(x_{2}\right), x_{2}\right) \in S$. Then $\left(x_{1}, \phi_{1}\left(x_{1}\right)\right) \in S$ and $\left(\phi_{2}\left(\phi_{1}\left(x_{1}\right)\right), \phi_{1}\left(x_{1}\right)\right) \in S$ and so $x_{1} \sim \phi_{2}\left(\phi_{1}\left(x_{1}\right)\right)$ for almost all $x$ and similarly $x_{2} \sim \phi_{1}\left(\phi_{2}\left(x_{2}\right)\right)$. But this means precisely that $R_{1}$ and $R_{2}$ are Mackey equivalent in the sense of Theorem 3 of FMI; and as they are both infinite, they are, by the Corollary of Theorem 3 of FMI, isomorphic by means of a map $\gamma$ with the property that $\gamma(x) \sim \phi_{1}(x)$. This says that $\Gamma(\phi) \subset S$ as desired.

Just as in the proof of Theorem 1 we obtain that the algebra $\mathbf{D}$ has uniform multiplicity so that $\mathscr{K}=L^{2}\left(S, \lambda, \mathcal{K}_{0}\right)$ for a Hilbert space $\mathcal{K}_{0}$. $\mathscr{K}_{0}$ of course will turn out to be one dimensional so that $\mathbf{D}$ is maximal abelian on $\mathcal{H}$. Let us further select $\lambda$ so that its disintegration with respect to projection to $X_{1}, \lambda_{x}$ are counting measures on $X_{2}$; that is, $\lambda_{x}$ gives each point in $R_{2}(\gamma(x))$ mass one where $\gamma$ is the fixed isomorphism of $R_{1}$ onto $R_{2}$ supplied by Proposition 5.5.

Now let $\phi_{2}$ be an element of $\mathscr{H}=L^{2}\left(S, \lambda, \mathcal{H}_{0}\right)$ which vanishes off $\Gamma(\phi)$ and with $\phi_{2}(x, \gamma(x))$ a unit vector in $\mathcal{H}_{0}$.

Proposition 5.6. The vector $\phi_{2}$ is separating and cyclic for $\mathbf{M}$.

Proof. We show that it is cyclic for $\mathbf{M}$ and $\mathbf{M}^{\prime}$. Let $\mathcal{H}_{1}=\left(\mathbf{M} \cdot \phi_{2}\right)^{-}$and let $B \in J_{1} A_{1} J_{1}=B_{1} ; B$ corresponds to an element denoted by $b$ in $L^{\infty}\left(X_{1}\right)$ so that $\left(B \phi_{2}\right)(x, y)=b(x) \phi_{2}(x, y)$. But if we let $a(y)=b\left(\gamma^{-1}(y)\right) \in L^{\infty}\left(X_{2}\right), a$ represents an element $A$ of $A_{2}$ and

$$
\left(A \phi_{2}\right)(x, y)=a(y) \phi_{2}(x, y)=b\left(\gamma^{-1}(y)\right) \phi_{2}(x, y)=b(x) \phi_{2}(x, y)
$$

since $\phi_{2}(x, y)=0$ unless $x=\gamma^{-1}(y)$. Moreover, if $T \in \mathbf{M}$, and $B \in \mathbf{B}_{1}$, $B T\left(\phi_{2}\right)=T B\left(\phi_{2}\right)=T A\left(\phi_{2}\right) \in \mathbf{M} \phi_{2}$. Thus $\mathbf{M} \phi_{2}$ and hence its closure $\mathcal{H}_{1}$ is invariant under $\mathbf{B}_{1}$, and so the projection $P_{1}$ onto $\mathcal{K}_{1}$ commutes with $\mathbf{B}_{1}$. Since it also commutes with $\mathbf{M}$, it is maximal abelian in $\mathbf{M}^{\prime}$. Consequently $\left(P_{1} \psi\right)(x, y)=I_{E}(x)(x, y)$ where $I_{E}$ is the characteristic function of some set $E \subset X_{1}$. But since $P_{1} \phi_{2}=\phi_{2}$ and $\phi_{2}(x, \phi(x))$ is a unit vector, we must have 
$E=X_{1}$ a.e. so $\mathcal{T}_{1}=\mathscr{T}$ and $\phi_{2}$ is cyclic. A symmetric argument interchanging $B_{1}$ and $A_{2}$ shows that $\phi_{2}$ is also cyclic for $M^{\prime}$.

Now let us consider the vector state $\omega_{2}$ of $\mathbf{M}^{\prime}$ defined by $\omega_{2}(M)=$ $\left(M \phi_{2}, \phi_{2}\right)$. For $B \in J_{1} \mathrm{~A}_{1} J_{1}=\mathrm{B}_{1}$, and the corresponding function $b$,

$$
\begin{aligned}
\left(B \phi_{2}, \phi_{2}\right) & =\int b\left(x_{1}\right) \phi_{2}\left(x_{1}, x_{2}\right) d \lambda\left(x_{1}, x_{2}\right) \\
& =\int b\left(x_{1}\right)\left(\sum_{x_{2} \sim \gamma\left(x_{1}\right)}\left|\phi_{2}\left(x_{1}, x_{2}\right)\right|\right)^{2} d \mu_{1}\left(x_{1}\right) \\
& =\int b\left(x_{1}\right) d \mu_{1}\left(x_{1}\right)=\left(B \phi_{1}, \phi_{1}\right)=\omega_{1}(B)
\end{aligned}
$$

where $\phi_{1}$ is the separating and cyclic vector for $\mathbf{M}$ used in the construction of $\mathbf{M}=\mathbf{M}\left(R_{1}, s_{1}\right)$ and $\omega_{1}$ is the state it defines of $\mathbf{M}^{\prime}$. Now let $S_{2}, J_{2}, \Delta_{2}$ be the Tomita-Takesaki operators associated with the vector $\phi_{2}$ and $\mathbf{M}^{\prime}$.

Let $B \in \mathrm{B}_{1}$. Then $S_{2}\left(B \phi_{2}\right)=B^{*} \phi_{2}$. Also $S_{2}^{*}$ is defined by $S_{2}^{*}\left(A \phi_{2}\right)=A^{*} \phi_{2}$ for $A \in \mathbf{M}$ and hence for $A \in \mathrm{A}_{2}$. But we have already noted that $A\left(\phi_{2}\right)=$ $B\left(\phi_{2}\right)$ for an appropriate $B \in \mathbf{B}_{1}$. Thus $S_{2}\left(D \phi_{2}\right)=S_{2}^{*}\left(D \phi_{2}\right)=\bar{D} \phi_{2}$ and it follows that $\Delta_{2}^{1 / 2}\left(D \phi_{2}\right)=D \phi_{2}$ for $D \in D$, and so $\Delta_{2}^{i t}\left(D \phi_{2}\right)=D \phi_{2}$. Then $\Delta_{2}^{i t} B \Delta_{2}^{i t} \phi_{2}=\Delta_{2}^{i t} B \phi_{2}=B \phi_{2}$ for $B \in \mathbf{B}_{1}$ and since $\phi_{2}$ is separating, $\Delta_{2}^{i t} B \Delta_{2}^{i t}=B$.

It follows from Takesaki [1] that there is a faithful normal conditional expectation $E_{2}$ from $\mathbf{M}^{\prime}$ onto $\mathbf{B}_{1}$ such that $\omega_{2}(T)=\omega_{2}\left(E_{2}(T)\right)$ for $t \in \mathbf{M}^{\prime}$. However, a conditional expectation from $\mathbf{M}^{\prime}$ onto $\mathbf{B}_{1}$ is unique if it exists (Tomiyama [1] and Connes [1]), and we already have one from the construction of $\mathbf{M}$ as $\mathbf{M}\left(R_{1}, s_{1}\right)$, namely $E=J E_{1} J$ where $E_{1}$ is the conditional expectation of $\mathbf{M}$ onto $\mathbf{A}_{1}$. Thus

$$
\omega_{1}(T)=\omega_{1}(E(T))=\omega_{2}(E(T))=\omega_{2}\left(E_{2}(T)\right)=\omega_{2}(T),
$$

and so the states $\omega_{1}$ and $\omega_{2}$ of $\mathbf{M}^{\prime}$ are the same. Since $\omega_{1}(T)=\left(T \phi_{1}, \phi_{1}\right)$ and $\omega_{2}(T)=\left(T \phi_{2}, \phi_{2}\right)$, it follows that there is a unitary $U$ in $\left(M^{\prime}\right)^{\prime}=M$ with $U \phi_{1}=\phi_{2}$.

But now we can characterize $A_{1}$, the diagonal subalgebra of $\mathbf{M}\left(R_{1}, s_{1}\right)$, as $\left\{A: A \in \mathbf{M}, A \phi_{1} \in \mathrm{B}_{1} \phi_{1}\right\}$ and the analysis above shows that $\mathrm{A}_{2} \subset\{A: A \in$ $\left.\mathbf{M}, A \phi_{2} \in \mathbf{B}_{1} \cdot \phi_{2}\right\}$ and since $\phi_{2}$ is separating, we have equality. But now from $U \phi_{1}=\phi_{2}$ it follows that $U A_{1} U^{-1}=A_{2}$.

There is a final fact about Cartan subalgebras and inner conjugacy which is worth noting.

TheOREM 10. If $\mathbf{A}_{1}$ and $\mathbf{A}_{2}$ are Cartan subalgebras of $\mathbf{M}$, then $\mathbf{A}_{1}$ and $\mathbf{A}_{2}$ are conjugate if and only if $\mathbf{A}_{1} \oplus \mathbf{A}_{2}$ is a Cartan subalgebra of $\mathfrak{T}_{2}(\mathbf{M})$, the two by two matrices over $\mathbf{M}$.

Proof. We note that $\mathbf{A}_{1} \oplus \mathbf{A}_{2}$ is maximal abelian in $\mathfrak{T}_{2}(\mathbf{M})$ and has a 
conditional expectation, namely $E\left(\left(M_{i j}\right)\right)=E_{1}\left(M_{11}\right) \oplus E_{2}\left(M_{22}\right)$ where $E_{1}$ and $E_{2}$ are the conditional expectations of $\mathbf{M}$ onto $\mathbf{A}_{1}$ and $\mathbf{A}_{2}$. It is clear that the normalizer of $\mathbf{A}=\mathbf{A}_{1} \oplus \mathbf{A}_{2}$ generates at least the block diagonal algebra $\mathbf{M} \oplus \mathbf{M}$. But $\mathbf{M} \oplus \mathbf{M}$ is maximal as a von Neumann subalgebra, as is easily seen, and so $\mathbf{A}_{1} \oplus \mathbf{A}_{2}$ is a Cartan subalgebra if and only if $\mathbf{N}(\mathbf{A})$ contains something which is not block diagonal.

Now if $\mathbf{A}_{1}$ and $\mathbf{A}_{2}$ are inner conjugate with $\mathbf{A}_{2}=U \mathbf{A}_{1} U^{*}$, it is clear that the matrix $V=\left(\begin{array}{cc}0 & U^{*} \\ U & 0\end{array}\right)$ is in the normalizer of $\mathbf{A}$, and we are done.

Conversely, let $\mathbf{N}(\mathbf{A})$ contain a non block diagonal matrix so that $\mathbf{A}$ is a Cartan subalgebra. Now $\mathbf{A} \cong L^{\infty}\left(X_{1} \cup X_{2}\right)$ where $\mathbf{A}_{i} \cong L^{\infty}\left(X_{i}\right)$ and then we obtain a relation $R$ on $\left(X_{1} \cup X_{2}\right) \times\left(X_{1} \cup X_{2}\right)$ which, in $X_{i} \times X_{i}$, is $R_{i}$. Let $S_{1}=R \cap\left(X_{1} \times X_{2}\right)$ and $S_{2}=R \cap\left(X_{2} \times X_{1}\right)$; the sets $S_{i}$ are just the type that occur in the proof of 'Theorem 9 . In fact, it follows immediately that $A_{1}$ is discrete over $\mathbf{A}_{2}$ and $\mathbf{A}_{2}$ is discrete over $\mathbf{A}_{1}$. Thus if $M$ is purely infinite, $\mathbf{A}_{1}$ and $A_{2}$ are inner conjugate by Theorem 9 . Then by direct integral theory we can, in fact, reduce to the case when $\mathbf{M}$ is a $\mathrm{II}_{1}$ factor. One can then prove that $S_{1}$ contains the graph of a Borel isomorphism (cf. Proposition 5.5) of $R_{1}$ onto $R_{2}$. The extra piece of information one uses is the comparison of projections $\mathfrak{T}_{2}(\mathbf{M})$. It is then fairly clear that $\mathbf{A}_{1}$ and $\mathbf{A}_{2}$ are inner conjugate. We omit further details as they are routine.

\section{REFERENCES}

L. Auslander and C. C. Moore [1], Unitary representations of solvable Lie groups, Mem. Amer. Math. Soc. No. 62 (1966), 199 pp. MR 34 \#7723.

A. Connes [1], Une classification des facteurs de type III, Ann. Sci. École Norm. Sup. (4) 6 (1973), 133-252. MR 49 \#5865.

, [2], Caractérisation des espaces vectoriels ordonnés sous-jacents aux algèbres de von Neumann, Ann. Inst. Fourier (Grenoble) 24 (1974), 121-155 (1975). MR 51 \# 13705.

$\# 6438$.

, [3], A factor not anti-isomorphic to itself, Ann. of Math. (2) 101 (1975), 536-554. MR 51

[4], Outer conjugacy classes of automorphisms of factors, Queen's Math. Preprint \# 1975-12, Queen's Univ., Kingston, Ont.

J. Dixmier [1], Sous-anneaux abéliens maximaux dans les facteurs de type fini, Ann. of Math. (2) 59 (1954), 279-286. MR 15, 539.

J. Feldman and C. C. Moore [1], Ergodic equivalence relations, cohomology, and von Neumann algebras. I., Trans. Amer. Math. Soc. 234 (1977), 289-324.

, [2], Ergodic equivalence relations, cohomology, and von Neumann algebras, Bull. Amer.

Math. Soc. 81 (1975), 921-924.

B. Fuglede and R. V. Kadison [1], On a conjecture of Murray and von Neumann, Proc. Nat. Acad. Sci. U.S.A. 37 (1951), 420-425. MR 13, 255.

P. Ghez, R. Lima and D. Testard [1], Une extension d'une théorème de A. Connes sur les facteurs constructibles, Comm. Math. Phys. 32 (1973), 305-311. MR 50 \#3808.

F. Greenleaf [1], Invariant means on topological groups and their applications, Van Nostrand Rheinhold, New York, 1969. MR 40 \# 4776.

P. Hahn [1], Haar measures and convolution algebras on ergodic groupoids, Thesis, Harvard Univ., 1975.

R. V. Kadison and J. R. Ringrose [1], Derivations and automorphisms of operator algebras, Comm. Math. Phys. 4 (1967), 32-63. MR 34 \#6552. 
W. Krieger [1], On constructing non-*-isomorphic hyperfinite factors of type III, J. Functional Analysis 6 (1970), 97-109. MR 41 \#4260.

[2], On ergodic flows and the isomorphism of factors, Math. Ann. 223 (1976), 19-70.

C. C. Moore [1], Group extensions and cohomology for locally compact groups. III, Trans. Amer. Math. Soc. 221 (1976), 1-34.

F. J. Murray and J. von Neumann [1], On rings of operators. IV, Ann. of Math. (2) 44 (1936), 116-229.

M. Rieffel and A. van Daele [1], The commutation theorem for tensor products of von Neumann algebras, Bull. London Math. Soc. 7 (1975), 257-260. MR 52 \#3977.

I. E. Segal [1], Abstract probability spaces and a theorem of Kolmogoroff, Amer. J. Math. 76 (1954), 721-732. MR 16, 149.

I. M. Singer [1], Automorphisms of finite factors, Amer. J. Math. 77 (1955), 117-133. MR 16, 597.

M. Takesaki [1], Conditional expectations in von Neumann algebras, J. Functional Analysis 9 (1972), 306-321. MR 46 \#2445.

J. Tomiyama [1], On some types of maximal abelian subalgebras, J. Functional Analysis 10 (1972), 373-386. MR 49 \#5876.

A. M. Veršik [1], Nonmeasurable decompositions, orbit theory, algebras of operators, Dokl. Akad. Nauk SSSR 199 (1971), 1004-1007 = Soviet Math. Dokl. 12 (1971), 1218-1222. MR 44 \#4537.

G. Zeller-Meier [1], Produits croisés d'une $C^{*}$-algèbre par un groupe d'automorphismes, J. Math. Pures Appl. (9) 47 (1968), 101-239. MR 39 \#3329.

Department of Mathematics, University of California, Berkeley, California 94720 